\title{
CNOT3 interacts with the Aurora B and MAPK/ ERK kinases to promote survival of differentiating mesendodermal progenitor cells
}

\author{
Moumita Sarkara,t,*, Matteo Martufia, ${ }^{\mathrm{t}}$, Monica Roman-Trufero ${ }^{a}$, Yi-Fang Wang $^{\mathrm{b}}$, Chad Whilding ${ }^{\mathrm{c}}$, \\ Dirk Dormannc, Pierangela Sabbattini ${ }^{a}$, and Niall Dillon ${ }^{a, d, *}$ \\ aGene Regulation and Chromatin Group, ${ }^{b}$ Bioinformatics and Computing, and cMicroscopy Facility, MRC London

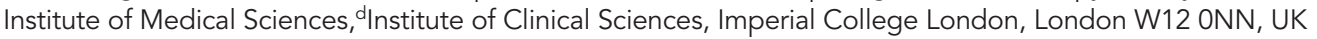

\begin{abstract}
Mesendoderm cells are key intermediate progenitors that form at the early primitive streak (PrS) and give rise to mesoderm and endoderm in the gastrulating embryo. We have identified an interaction between CNOT3 and the cell cycle kinase Aurora B that requires sequences in the NOT box domain of CNOT3 and regulates MAPK/ERK signaling during mesendoderm differentiation. Aurora B phosphorylates CNOT3 at two sites located close to a nuclear localization signal and promotes localization of CNOT3 to the nuclei of mouse embryonic stem cells (ESCs) and metastatic lung cancer cells. ESCs that have both sites mutated give rise to embryoid bodies that are largely devoid of mesoderm and endoderm and are composed mainly of cells with ectodermal characteristics. The mutant ESCs are also compromised in their ability to differentiate into mesendoderm in response to FGF2, BMP4, and Wnt3 due to reduced survival and proliferation of differentiating mesendoderm cells. We also show that the double mutation alters the balance of interaction of CNOT3 with Aurora B and with ERK and reduces phosphorylation of ERK in response to FGF2. Our results identify a potential adaptor function for CNOT3 that regulates the Ras/MEK/ERK pathway during embryogenesis.
\end{abstract}

\author{
Monitoring Editor \\ John Wallingford \\ University of Texas, Austin
}

Received: Feb 26, 2021

Revised: Sep 23, 2021

Accepted: Oct 1, 2021
This article was published online ahead of print in MBoC in Press (http://www .molbiolcell.org/cgi/doi/10.1091/mbc.E21-02-0089) on October 6, 2021.

TThese authors contributed equally to the study.

Author contributions: M.S., M.M., M.R.-T., and N.D. participated in the design of the study. M. S. identified and characterized the effects of the Cnot3 mutations on mesendodermal differentiation and ERK phosphorylation and the effect of Aurora $\mathrm{B}$ on nuclear localization of CNOT3 in lung cancer cells. M. M. carried out the CRISPR targeting in ESCs and the initial characterization of the nuclear localization of CNOT3 and binding of Aurora B to the NOT box Aurora B. M.R.-T. identified the Aurora B phosphorylation target sites in CNOT3 and provided expertise on CRISPR targeting. Y.-F.W. analyzed the RNA-seq data. C.W. and D.D. provided microscopy expertise and carried out the digital analysis of the PLA data. P.S. contributed to the phenotypic analysis of the mutant ESCs. M.S. and N.D. wrote the manuscript with contributions from M.M. and M.R.-T. N.D. supervised and coordinated the project.

*Address correspondence to: Moumita Sarkar (m.sarkar@imperial.ac.uk); Niall Dillon (niall.dillon@lms.mrc.ac.uk).

Abbreviations used: EB, embryoid body; ESC, embryonic stem cell; ME, mesendoderm; NLS, nuclear localization sequence; PLA, proximity ligation assay.

(c) 2021 Sarkar, Martufi, et al. This article is distributed by The American Society for Cell Biology under license from the author(s). Two months after publication it is available to the public under an Attribution-Noncommercial-Share Alike 4.0 International Creative Commons License (https://creativecommons.org/licenses/ by-nc-sa/4.0).

"ASCB®," "The American Society for Cell Biology $\bigotimes^{\circledR}$," and "Molecular Biology of the Cell@" are registered trademarks of The American Society for Cell Biology.

\section{INTRODUCTION}

Gastrulation in mammals and birds is initiated by differentiation of mesendoderm (ME) cells, which form at the primitive streak ( $\mathrm{PrS}$ ) as transient precursors expressing both mesodermal and endodermal markers in response to signaling from the visceral endoderm (VE) (Chuai et al., 2012; Stern and Downs, 2012; Wolfe and Downs, 2014; Costello et al., 2015; Wang and Chen, 2016). Ingression of ME cells along the midline of the PrS during gastrulation is accompanied by differentiation into mesoderm and definitive endoderm and formation of the embryonic germ layers. Signaling ligands that have been shown to be involved in specifying ME differentiation and PrS formation in the early embryo include Wnt3, activin, BMP4, and FGF2 (Tremblay et al., 2000; Fujiwara et al., 2002; Ben-Haim et al., 2006; Bernardo et al., 2011; Tsakiridis et al., 2014). In combination, these ligands can be used to induce embryonic stem cell (ESC) differentiation into ME, which is characterized by expression of the mesodermal marker Brachury and endodermal markers GATA4, GATA6, and FOXA2 (reviewed by Sui et al., 2013).

The RAS/MEK/ERK signaling pathway regulates an extremely diverse range of cellular processes at all stages of development 
(Roux and Blenis, 2004) and has been shown to play an essential role in the formation of ME in mouse and human ESCs (Yu et al., 2011; Wang and Chen, 2016). RAS is activated in response to binding of growth factors such as FGF and EGF to their cognate receptors, triggering a cascade composed of RAF, MEK, and ERK that is critical for growth, survival, and differentiation of a wide range of cell types (Roux and Blenis, 2004; Krens et al., 2006). Signaling through this pathway has a well-established involvement in promoting cell survival via up-regulation of expression of antiapoptotic proteins such as BCL2 (Deng et al., 2000; Subramanian and Shaha, 2007). This occurs through ERK-mediated phosphorylation of RSK, which, in turn, phosphorylates and activates CAMP-response element binding protein (CREB) (Xing et al., 1998). Binding of CREB up-regulates $\mathrm{Bcl} 2$ and other genes that prevent apoptosis (Wang et al., 2003). ERK also phosphorylates and stabilizes the Bcl2 family member Mcl1, enhancing its antiapoptotic activity (Domina et al., 2004). ERK2 null embryos fail to form mesoderm and exhibit increased apoptosis (Yao et al., 2003).

The diversity of functional roles for MAPK/ERK signaling implies the existence of multiple secondary levels of regulation that direct the signals toward their different targets. Adaptor proteins that bind more than one signaling component are one type of mechanism that can be used to regulate MAPK/ERK activity and channel it toward specific targets in a given cell type (Kolch, 2005; Sacks, 2006). Several adaptor proteins have been identified that affect ERK activation including IQGAP1 and kinase suppressor of Ras-1 (KSR), both of which bind the MEK and ERK kinases (Roy et al., 2004, 2005).

CNOT3 was first identified as a component of the CCR4-Not complex, which is involved in regulating transcription and RNA processing in the nucleus and mRNA turnover in the cytoplasm (Collart et al., 2013). Studies in ESCs have shown that CNOT3 acts in conjunction with the transcription factors CMYC and ZFX to form part of a transcriptional regulatory module that binds to a number of gene promoters in ESCs (Hu et al., 2009) and has been reported to inhibit differentiation of ESCs into extraembryonic lineages (Hu et al., 2009; Zheng et al., 2012). Knockout of Cnot3 in mice leads to embryonic lethality at the blastocyst stage caused by loss of inner cell mass cells (Neely et al., 2010).

In addition to its early embryonic functions, CNOT3 has been shown to have important roles in B-lymphopoesis and maintenance of adipose tissues in adult mice (Yang et al., 2016; Li et al., 2017). Cnot3 has also been shown to have tumor suppressor and tumorpromoting properties (De Keersmaecker et al., 2013; Cejas et al., 2017; Shirai et al., 2019). Mutations that alter the protein sequence have been identified in a range of cancers, with the largest number observed in prostate and colon cancers.(Forbes et al., 2017; Priestley et al., 2019). The diverse biological and cellular roles of CNOT3 and the presence of the C-terminal specialist protein-protein interaction domain, the NOT box, and a C-terminal coiled-coil domain, linked by a long central domain that has the characteristics of an intrinsically disordered region (Boland et al., 2013), suggest that it could function as an adaptor that mediates cross-talk between different cellular pathways.

Here we show that CNOT3 has the properties of an adaptor protein that links the cell cycle kinase Aurora B with the MAPK/ERK signaling pathway. Aurora B and ERK interact with the NOT box domain of CNOT3, and phosphorylation of the protein by Aurora B at sites adjacent to a nuclear localization sequence (NLS) promotes localization of CNOT3 to the nucleus and increases interaction between ERK and CNOT3. Mutation of the Aurora B target sites reduces the level of active phosphorylated ERK in ESCs, preventing efficient differentiation of ME in response to FGF. We also present evidence supporting involvement of Aurora B-mediated phosphorylation and nuclear localization of CNOT3 in the tumorigenic effects of CNOT3.

\section{RESULTS}

\section{Phosphorylation by Aurora B localizes CNOT3 to ESC nuclei}

The Aurora B cell cycle kinase phosphorylates multiple protein targets during mitosis and cytokinesis as part of the chromosomal passenger complex (reviewed by Carmena et al., 2012) and is also involved in regulating the G1 to S-phase transition (Song et al., 2007; Trakala et al., 2013). Up-regulation of Aurora B protein levels has been shown to be a marker for lymph node metastasis in lung, colon, and breast cancers (Tuncel et al., 2012; Takeshita et al., 2013; Zhang et al., 2015). We initially identified a strong interaction between Aurora B and CNOT3 in a coimmunoprecipitation screen and analysis of primary resting and activated mouse B-lymphocytes (Frangini, 2013) (M. Martufi and N. Dillon, unpublished data). Because of the known role of CNOT3 in ESC pluriotency and lineage choice (Hu et al., 2009; Zheng et al., 2012), we tested whether it interacts with Aurora B in ESCs. Coimmunoprecipitation of ESC extracts with anti-Aurora B antibody showed that CNOT3 and Aurora $B$ interact strongly in ESCs (Figure 1A).

Direct interaction of specific regions of CNOT3 with Aurora $B$ was demonstrated by using GST-tagged Aurora B for in vitro pull down of ${ }^{35} \mathrm{~S}$-labeled wild-type and CNOT3 deletion mutant proteins (Figure 1, B and C; Supplemental Figure S1A). The results of the deletion analysis showed that deletion of the entire C-terminal NOT box domain ( $\Delta 651-751)$ results in near-complete loss of Aurora B binding. Deletion of the $\mathrm{N}$-terminal segment of the NOT box $(\Delta 651-700)$ had no effect on binding of Aurora $B$, whereas deletion of the C-terminal region of the NOT box $(\Delta 701-751)$ strongly increased the amount of interaction observed (Figure 1C, bottom panels). Deletion of the region of CNOT3 encompassing the 200 bases at the $\mathrm{N}$-terminal end of the protein (see Figure 1C, $\Delta 1-200$ deletion mutant) also resulted in reduced binding. These results indicate a complex interaction between Aurora B and CNOT3, with binding dependent on the NOT box domain, but with other regions involved in determining the level of interaction.

In vitro phosphorylation of CNOT3 by Aurora B, followed by mass spectrometry analysis, revealed phosphorylation at residues T292 and S294 in the CNOT3 protein (Supplemental Figure S2A). T292 is located in a consensus Aurora B phosphorylation sequence $\left({ }^{290} \mathrm{R}-\mathrm{S}-\mathrm{T}^{292}\right)$ (Figure 1B). Phosphorylation of purified in vitro translated wild-type and mutant CNOT3 proteins by Aurora B in the presence of [ $\left.\gamma^{32} \mathrm{P}\right]-A T P$ showed reduced labeling of a double CNOT3T292A/S294A mutant protein (Figure 1D). A reduction in labeling was also observed when T292 alone was mutated to A, but the effect was less pronounced when S294 was mutated on its own. Deletion of the NOT box region abolished labeling, indicating that docking of Aurora B on the NOT box is required for the kinase to phosphorylate CNOT3 (Figure 1D). It should be noted that mutation of T292/S294 does not completely eliminate in vitro phosphorylation, implying that one or both of two Aurora B consensus sites in the protein amino-terminal and C-terminal regions of the protein (Supplemental Figure S1A) are also targets for Aurora B when it binds to the NOT box.

Inspection of publicly available high-throughput mass spectrometry data from human tissues (Hornbeck et al., 2015) revealed that the T292/S294 residues are part of a phosphorylation hotspot extending from residues 291 to 299 of CNOT3 (Supplemental Figure S1B). The phosphorylation hotspot, which includes T292 and S294, is located adjacent to a sequence motif ( ${ }^{286} \mathrm{~K}-\mathrm{K}-\mathrm{R}-\mathrm{G}-\mathrm{R}^{290}$ ) (Figure 1B; 
A

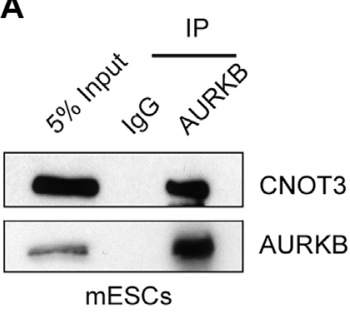

C

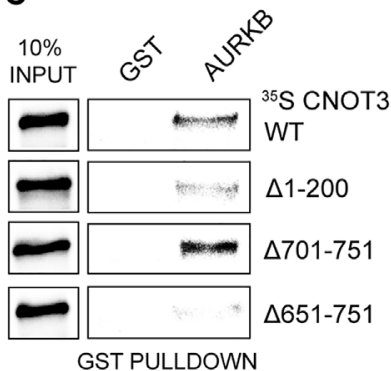

GST PULLDOWN
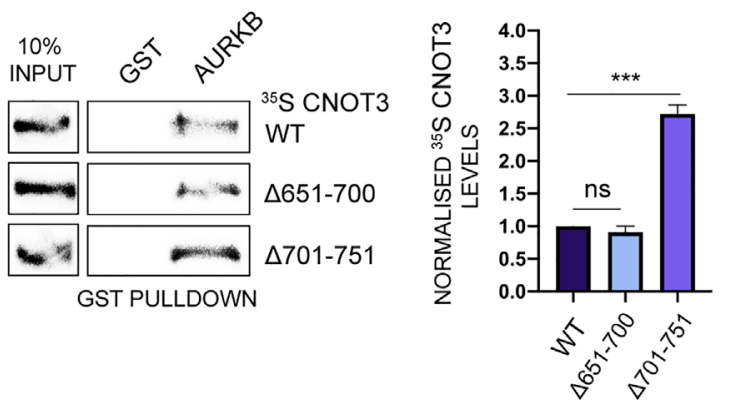

E

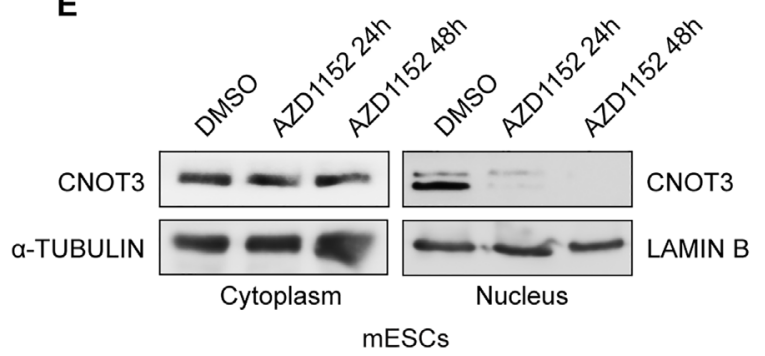

B
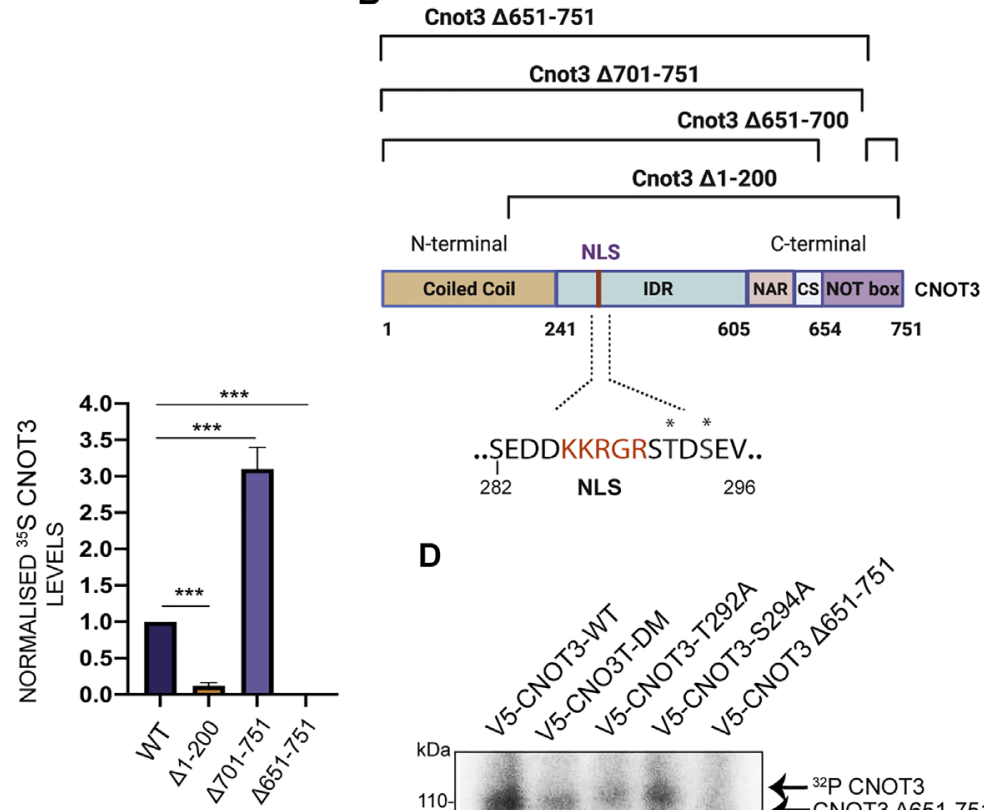

D

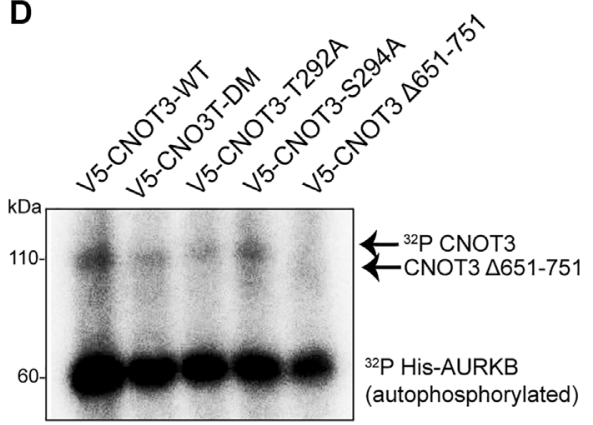

In vitro kinase assay

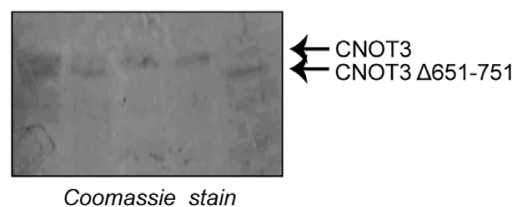

$\mathbf{F}$

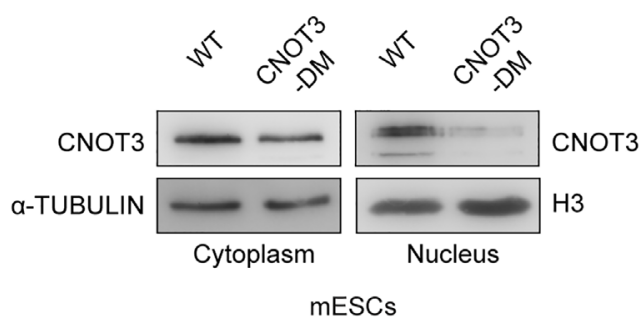

FIGURE 1: Phosphorylation by Aurora B increases nuclear localization of CNOT3. (A) Representative IP carried out on cell extracts prepared from wild-type ESCs using anti-Aurora B antibody (AURKB). Blots were probed with anti-CNOT3 and anti-Aurora B. Immunoglobulin G $(\mathrm{lgG})=$ control IP with mouse lgG. Input $=5 \%$ of the extract. (B) Schematic representation of the domain organization of CNOT3 (Boland et al., 2013) and the Cnot3 deletion mutants used for GST pull downs. IDR = intrinsically disordered region; NAR = NOT1 anchor region; CS = connector sequence; NLS (red type) = nuclear localization sequence. The Aurora B consensus phosphorylation site is underlined. (C) In vitro GST pull-down assay using GST-Aurora B and ${ }^{35}$ S-labeled in vitro transcribed/translated wild-type (WT) CNOT3 and CNOT3 deletion mutants (as shown in B). Top and bottom left panels: Representative pull downs of GST-Aurora B and ${ }^{35} \mathrm{~S}$ labeled WT CNOT3 and CNOT3 deletion mutants. GST-only was used as a negative control for each pull down. Input $=10 \%$ of labeled proteins. Right panels: Histograms show quantification of band intensities normalized to input and calculated relative to WT-CNOT3. Mean $\pm \mathrm{SEM} ; n=3$ (unpaired $t$ test; ${ }^{* \star \star} P<0.001$ ). (D) Top panel: In vitro kinase assay (top) using $\gamma-32$ P-ATP on purified V5-tagged in vitro transcribed/translated WT CNOT3, and single mutant (CNOT3-T292A or CNOT3-S294A), double mutant (CNOT3-DM), and CNOT3 $\triangle 651-751$. AURKB = autophosphorylated Aurora B. Bottom panel: Coomassie stain showing protein levels. (E) Representative immunoblot of CNOT3 from cytoplasmic and nuclear extracts of WT ESCs, treated with AZD1152 for 24 and 48 h. Dimethyl sulfoxide (DMSO): vehicle control. Cytoplasmic loading control for $E$ and F: $\alpha$-tubulin. Nuclear loading control for $E$ and F: lamin B. (F) Representative immunoblot analysis of CNOT3 levels in cytoplasmic and nuclear extracts of wild-type (WT) and Cnot3-DM ESCs. 

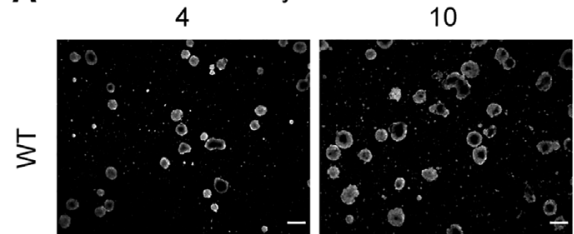
Days
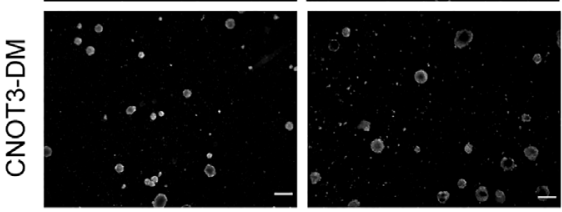

B

C DAPI BRACHURY
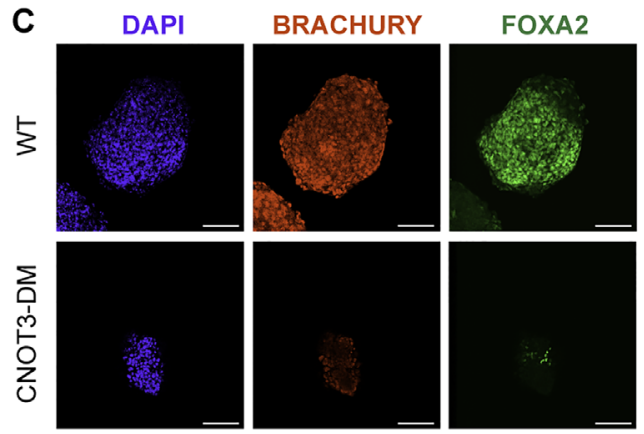

MERGED
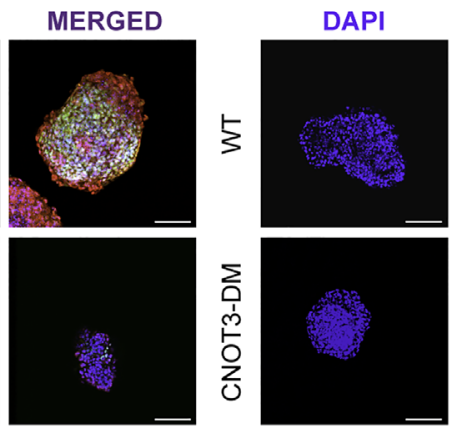

NESTIN

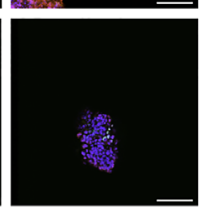

Number of EBs
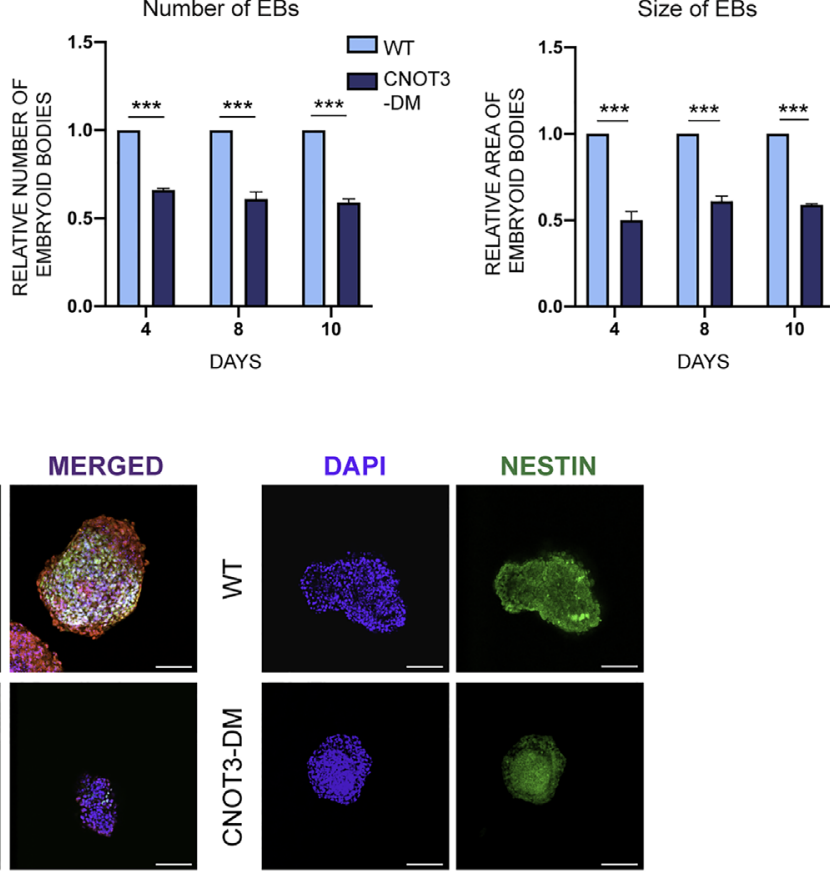

FIGURE 2: Mutation of CNOT3-T292A/S294 affects EB germ layer formation. (A) Representative phase-contrast images of EBs formed from wild-type (WT) and Cnot3-DM ESCs after 4 and $10 \mathrm{~d}$. Scale bar $=100 \mu \mathrm{m}$. (B) Histograms show the number (left panel) and size, assessed by measuring maximum diameter (right panel), of EBs formed from Cnot3-DM ESCs relative to WT cells at 4,8 , and $10 \mathrm{~d}$, respectively. Mean \pm SEM ( $t$ test; ${ }^{\star \star \star} P<0.001 ; n=3$ ). (C) Left panel: Representative confocal images of EBs derived from WT and Cnot3-DM ESCs stained with Brachury (red) and FOXA2 (green) at $8 \mathrm{~d}$ (scale bar $=100 \mu \mathrm{m})$. Right panel: Staining of WT and Cnot3-DM EBs for nestin (green) (scale bar = $100 \mu \mathrm{m})$. Nuclei were stained with DAPI. Staining for OTX2 is shown in Supplemental Figure S3A.

Supplemental Figure S1A) that has been shown to form part of a functional NLS in the Toxoplasma gondii GCN5-B histone acetyl transferase and the influenza D virus nucleoprotein (Dixon et al., 2011; Donchet et al., 2019; Tang et al., 2019), suggesting that phosphorylation of this region could be involved in nuclear localization. To directly test this idea, ESCs were incubated for 24 and $48 \mathrm{~h}$ with the specific Aurora B inhibitor AZD1152. Comparison of the levels of CNOT3 in the nuclear and cytoplasmic fractions after $24 \mathrm{~h}$ incubation with AZD1152 showed a substantial reduction in the amount of CNOT3 in the nucleus (Figure 1E), and the level was further reduced after $48 \mathrm{~h}$ of treatment. The level of CNOT3 in the cytoplasm was largely unaffected (Figure 1E), and inhibition of Aurora B did not substantially affect the cell cycle of the ESCs (Supplemental Figure S2, C and E). These results provide evidence that phosphorylation of CNOT3 by Aurora $B$ is involved in specifying localization of CNOT3 to the ESC nucleus. We confirmed this by transfecting V5tagged CNOT3 expression constructs carrying mutations of either T292 or S294, or of both residues, to alanine, into HEK293 cells. The results showed reduction of nuclear CNOT3 for each of the single mutants and the double mutation, indicating that both Aurora B target sites contribute to nuclear localization in this assay (Supplemental Figure S2F).

CRISPR/Cas9 mutagenesis was used to simultaneously mutate the T292 and S294 residues to alanine in mouse ESCs (Supplemental Figure S2B). Generation of three ESC clones that were homozygous for the T292A/S294A double mutation (Cnot3-DM) was confirmed by sequencing (Supplemental Figure S2B). The mutant ESCs grew normally and had the characteristic cell cycle profile of ESCs (Supplemental Figure S2, D and E). Analysis of nuclear CNOT3 levels showed a substantial reduction in the amount of CNOT3 in the nuclei of Cnot3-DM ESCs compared with WT cells (Figure 1F). Cytoplasmic levels of CNOT3 were largely unchanged in the mutant cells.

\section{Effect of mutating CNOT3-T292/S294 on embryoid body formation}

To test whether the absence of phosphorylation of CNOT3-T292/ S294 affects ESC pluripotency and differentiation potential, wildtype and Cnot3-DM ESCs were cultured under conditions that promoted the formation of embryoid bodies (EBs) (see Materials and Methods for details). The results show that the double Cnot3 mutation (Cnot3-DM) resulted in a 40\% reduction in EB numbers after 10 $d$ in culture, and the average size of the EBs was reduced by $40 \%-$ $50 \%$ (Figure 2, A and B). Formation of the germ layers was analyzed by staining the EBs for the lineage markers Brachyury, FOXA2, and Nestin. Brachyury is expressed in mesoderm, and FOXA2 is expressed mainly in endoderm but is also present in axial mesoderm, which gives rise to the notochord (Burtscher and Lickert, 2009). Nestin is a marker for ectoderm. All three markers were strongly expressed in EBs formed from wild-type ESCs, with Brachyury giving broad staining of the central and peripheral regions of the EBs, whereas FOXA2 staining was more restricted to the central regions (Figure 2C). The ectodermal Nestin staining was broadly distributed in the EBs but was strongest at the periphery. In contrast, the Cnot3DM EBs showed very low staining for Brachyury and FOXA2 and strong central and peripheral staining for Nestin (Figure 2C). The EBs were also stained for OTX2 and showed a broad staining pattern, similar to Nestin (Supplemental Figure S3A). OTX2 is 
expressed widely in the germ layers during the early stages of gastrulation and then becomes restricted to anterior neuroectoderm (Simeone et al., 1993; Simeone, 1998). The strong costaining for OTX2 and Nestin in the mutant EBs and the near absence of Brachyury- and FOXA2-positive cells suggests that blocking phosphorylation of CNOT3-T292/S294 interferes with formation of mesodermal and endodermal germ layers, resulting in a predominance of ectodermal lineages.

\section{CNOT3-T292/S294 phosphorylation promotes ME differentiation and survival}

Germ layer formation during gastrulation involves a transition through an intermediate ME progenitor cell stage, which has been shown to depend on synergy between nodal/activin, BMP, Wnt, and FGF signaling pathways (Bakre et al., 2007; Willems and Leyns, 2008; Vallier et al., 2009; Yu et al., 2011; Wang and Chen, 2016). We carried out differentiations of wild-type and Cnot3-DM ESCs in a defined medium containing BMP4 and FGF2 and different combinations of activin, Wnt, and the GSK3 $\beta$ inhibitor CHIR99021, which stabilizes $\beta$-catenin, bypassing Wnt activation (Alexanian et al., 2017) (Figure 3, A-C) (see Materials and Methods). When applied to wild-type cells, incubation in the defined medium resulted in high levels of cell death for the first $2 \mathrm{~d}$ of incubation followed by gradual and then rapid expansion of ME cells. All of the combinations that included BMP4 and FGF2 and either Wnt, activin, or CHIR99021 gave rise to significant numbers of $\mathrm{ME}$ cells (Figure $3, \mathrm{~A}$ and $\mathrm{B}$ ), which comprised $80 \%-90 \%$ of the cultures based on staining for Brachyury and FOXA2 (Supplemental Figure S3C). Incubation with FGF2 and BMP4 alone also promoted ME differentiation of wildtype ESCs, but the total number of cells in the cultures and the number of differentiated cells were lower than for cultures containing activin or Wnt. The ME identity of the cells was confirmed by analysis of mRNA levels for mesodermal and endodermal lineage markers by quantitative RT-PCR (Supplemental Figure S3D). Staining for additional mesodermal and endodermal lineage markers (SMA: mesoderm; GATA4: endoderm) after 4 and $8 \mathrm{~d}$ of incubation with BMP4 + FGF2 provided further confirmation of ME differentiation (Supplemental Figure S4A and S4B).

Analysis of the Cnot3-DM cells showed that they gave significantly reduced numbers of differentiated cells for all ligand combinations tested (Figure 3, A and B), with the largest reductions relative to wild type observed with Wnt + FGF2 + BMP4 (80\%) and FGF2 + BMP4 (65\%). The reduction was confirmed by digital quantification of the number of Brachyury/FOXA2 and Brachyury/GATA4 double positive cells following differentiation with FGF2 + BMP4 (Figure 3D). The results of the analysis showed that the number of double positive cells for both marker combinations was dramatically reduced in the Cnot3-DM differentiations. Quantification of the total number of cells in the four and eight day differentiations of wild-type and Cnot3-DM ES cells showed that approximately $90 \%$ of the cells were positive for Brachyury and FOXA2 (Supplemental Figure S3C). Interestingly, the digital analysis also showed that, despite their reduced numbers, individual surviving Cnot3-DM cells expressed mesodermal and endodermal markers at levels comparable to those of wild-type cells (Figure 3E), suggesting that the primary defect was in cell survival. This was confirmed by Western blotting, which showed similar levels of mesodermal and endodermal markers in the wildtype and mutant cells Supplemental (Figure S4D).

Incubation of wild-type cells with activin on its own gave reduced survival compared with incubation with FGF2 + BMP4, whereas survival of the mutant cells was similar under both conditions (Supplemental Figure S3B). In contrast, cells that were incubated with Wnt in the absence of the other signaling ligands showed a reduction of around 10-fold in survival of wild-type cells and an even greater reduction for the Cnot3-DM cells (Supplemental Figure S3B). This result is consistent with FGF2 + BMP4 having a major role in promoting survival of the differentiating ME cells.

A time course from day 1 to day 8 of ME differentiation in the presence of BMP4 + FGF2 only showed reduced numbers of Cnot3DM cells from day 2 onward compared with wild-type cells (Figure $4, A$ and B). Digital analysis of cell differentiations at 2, 4, and $8 d$ of differentiation also showed that expression of SMA, FOXA2, GATA4, and Brachyury in individual Cnot3-DM cells is similar to that of wildtype cells (Supplemental Figures S4C and S5D), confirming that the major effect of the double mutation is on survival of differentiated $\mathrm{ME}$ cells rather than differentiation per se. Analysis of the differentiation capacity of the other two Cnot3-T292A/S294A mutant clones that were generated by the CRISPR/Cas9 targeting showed a similar failure to expand and proliferate in response to FGF2 + BMP4 (Supplemental Figure S5B), confirming that the effect was caused by the double mutation.

We also tested differentiation of the Cnot3-DM cells using classical protocols that induce differentiation into ectoderm, mesoderm, and endoderm as opposed to inducing a transitional ME stage. We found that differentiation into ectoderm by incubation in neurobasal medium (Sladitschek and Neveu, 2019) and into mesoderm using activin (Waese and Stanford, 2011) were both unaffected by the double mutation. However, differentiation of endoderm induced by FGF2 and retinoic acid (Kim et al., 2010b) showed a reduction of around $50 \%$ in the mutant cells (Supplemental Figure S5A). These findings support the idea that the regulatory role of CNOT3-T292/ S294 phosphorylation in ME differentiation and survival is linked to specific signaling pathways and suggest that FGF signaling is one of these pathways.

The conclusion that survival and proliferation of ME cells is strongly affected by the double mutation was further reinforced by the results of a time-lapse analysis of the differentiating wild-type and mutant cells between 4 and $8 d$ of ME differentiation (Supplemental Video 1). The time-lapse analysis showed an explosive proliferation of the wild-type cells, whereas the Cnot3-DM cells failed to expand and appeared to undergo high rates of cell death following cell division. The cell cycle profiles of the wild-type and mutant cells after $4 \mathrm{~d}$ of differentiation were similar, implying that the major effect of the mutation was on survival of differentiating ME cells (Supplemental Figure $\mathrm{S} 5 \mathrm{C}$ ).

Susceptibility of the Cnot3-DM cells to apoptosis during ME differentiation in response to FGF2 and BMP4 was directly assessed by staining wild-type and mutant cells with the apoptotic marker Annexin $\mathrm{V}$ and also by measuring propidium iodide (PI) uptake after 4 $\mathrm{d}$ of differentiation. The wild-type cells showed almost no staining for either cell death indicator, whereas the 4-d differentiated mutant cells were strongly stained for PI and Annexin V, indicating that the reduced number of differentiated mutant ME cells was due to high rates of apoptosis (Figure $4 \mathrm{C}$ ). This result suggests a functional relationship between CNOT3 phosphorylation and survival signals mediated by incubation with FGF2 and BMP4.

Further support for the conclusion that BMP4 + FGF2 promote survival of the differentiating ME cells was provided by a transcriptomic comparison of the wild-type and Cnot3-DM cells using RNAseq (Supplemental Figure S6, A and B). The analysis showed that 153 genes were significantly up-regulated and 155 were down-regulated in the mutant cells (Supplemental Table S3, A and B). Gene Ontology and GSEA analysis revealed that the mutation resulted in increased expression of genes that are associated with cell death 
BMP4+FGF2 +

WNT3 ACTIVINA +

CHIR99021
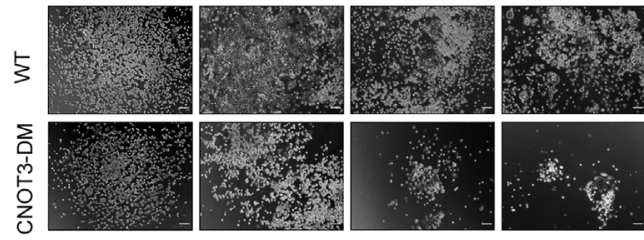

B

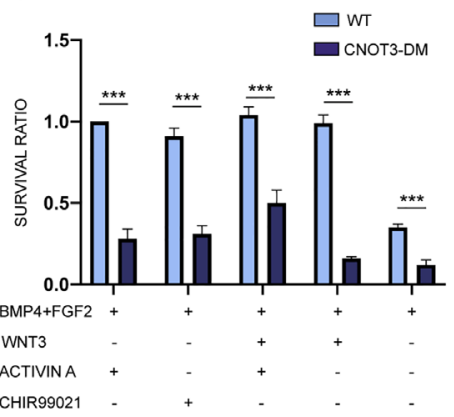

C

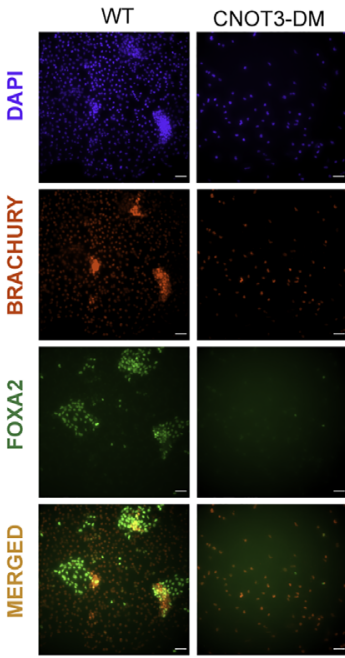

BMP4+FGF2+ACTIVIN A

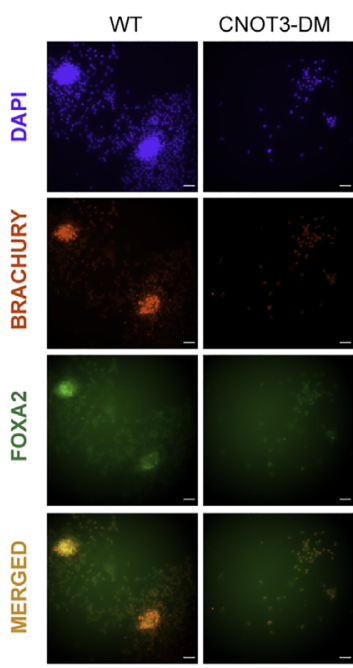

BMP4+FGF2+WNT3

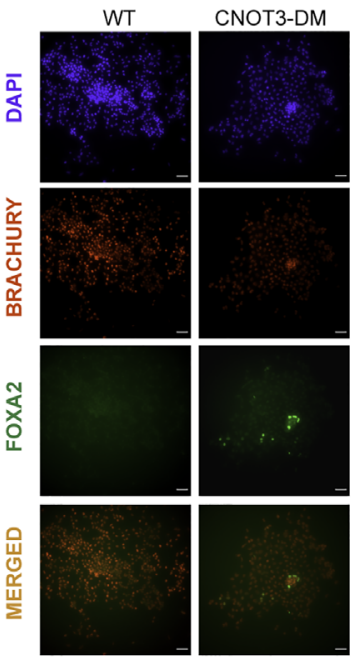

BMP4+FGF2+CHIR99021

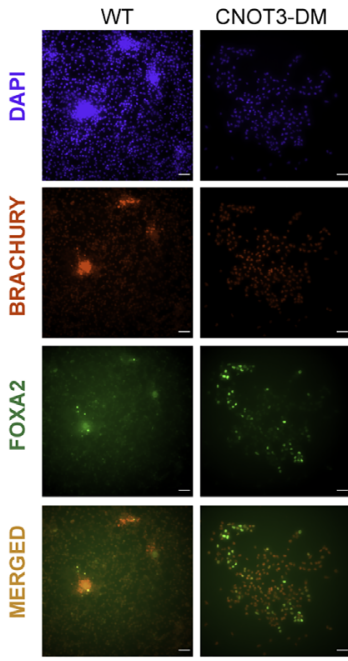

BMP4+FGF2+WNT3+ACTIVIN A

D

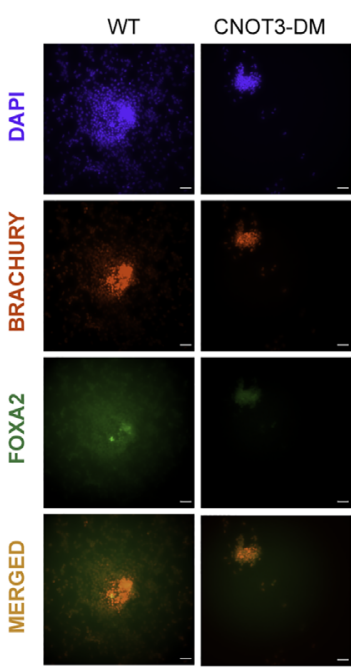

BMP4+FGF2

$E$

WNT3
4 days mesendoderm differentiation
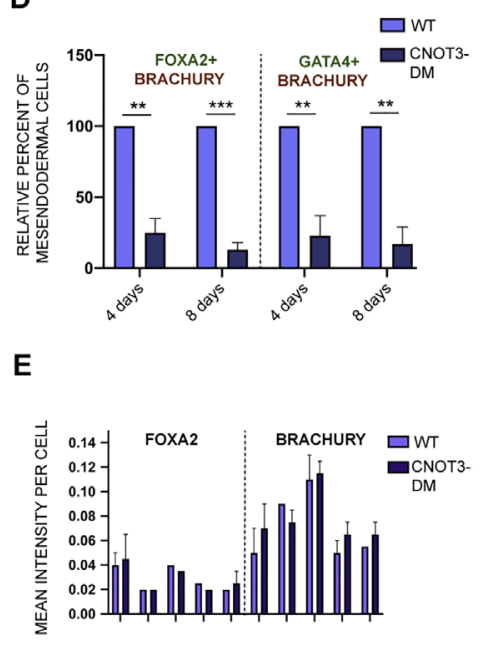

ACTIVINA

CHIR99021 and apoptosis (Supplemental Figure S6, C and $\mathrm{E})$. In addition, genes involved in cell proliferation and the MAPK cascade were down-regulated in the Cnot3-DM cells (Supplemental Figure S6D). The RNA-seq analysis also showed that the level of mRNA for FGFR1, which is the major FGF receptor that promotes ME differentiation (Deng et al., 1994; Yamaguchi et al., 1994; Ciruna and Rossant, 2001), was unchanged in the Cnot3-DM cells.

\section{Mutation of CNOT3-T292/S294}

reduces ERK phosphorylation

ERK1/2 phosphorylation by MEK is one of the key events in the response to stimulation by FGF (Roux and Blenis, 2004). ERK has been reported to up-regulate Aurora B expression in melanoma cells (Bonet et al., 2012), and phosphorylated ERK plays important roles in cell survival (Mebratu and Tesfaigzi, 2009). Involvement of the FGF/ MEK/ERK signaling pathway in promoting survival of cells during ME differentiation was supported by the observation that treatment of the cells with FGFR and MEK inhibitors caused a dramatic reduction in cell numbers (Figure 5A).

To assess the effect of the Cnot3 T292A/ S294A double mutation on ERK phosphorylation, extracts from wild-type and mutant ESCs that had been differentiated with FGF2 and BMP4 for $4 d$ were analysed by

or Wnt only is shown in Supplemental Figure S3B. (C) Representative immunofluorescence images of Brachury (red) and FOXA2 (green) following ME differentiation of WT and Cnot3-DM ESCs for $4 \mathrm{~d}$ induced by combinations of BMP4 + FGF2, activin A, CHIR99021, and Wnt3 (as indicated). Merged red and green images show ME cells. Nuclei were stained with DAPI; scale bar $=100 \mu \mathrm{m}$.

(D) Histogram shows percentage of

Brachyury-FOXA2 and Brachyury-GATA4 double positive cells in the WT and Cnot3DM ESCs treated with with BMP4 + FGF2 for 4 and $8 \mathrm{~d}$ of ME differentiation as in $\mathrm{C}$. Double positive cells were quantified from randomly chosen fields from at least 100 cells for each biological replicate and plotted as percent of the total number of nuclei (DAPI) relative to WT. (E) Histogram shows the expression of Brachury and FOXA2 in the WT and Cnot3-DM ESCs treated with different combinations of ligands used in C over $4 \mathrm{~d}$ of ME differentiation. The expression of each marker is plotted as mean intensity per cell and was quantified from randomly chosen fields for at least 100 cells for each biological replicate; $n=2$. All differences nonsignificant by unpaired $t$ test. 
A
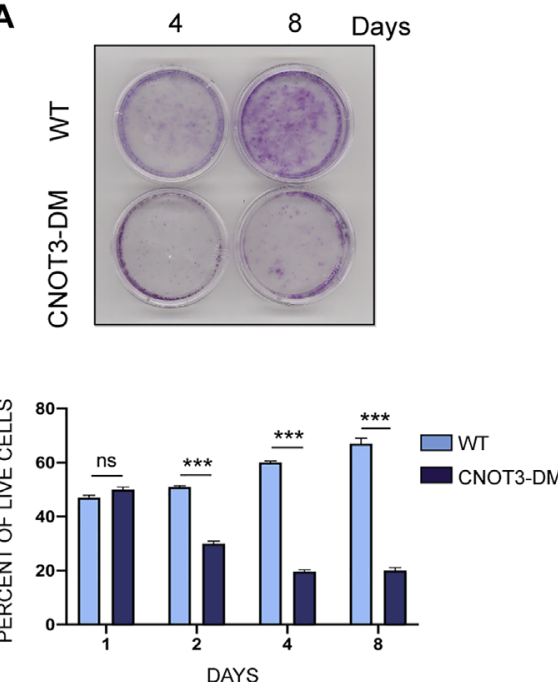

B
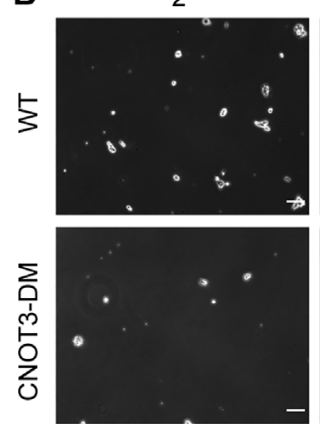
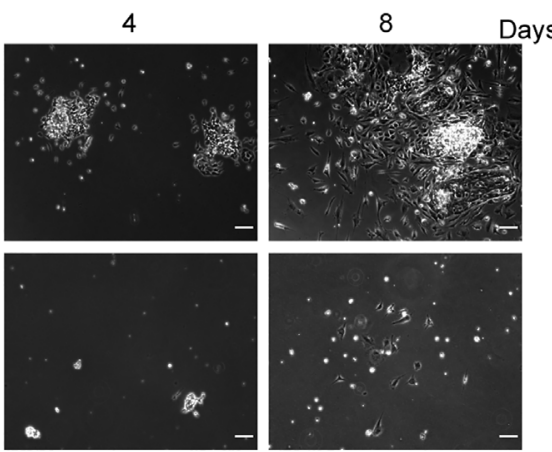

C

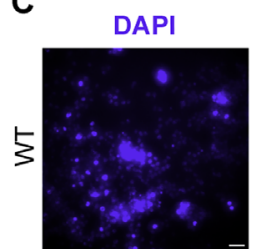

Annexin VIPI
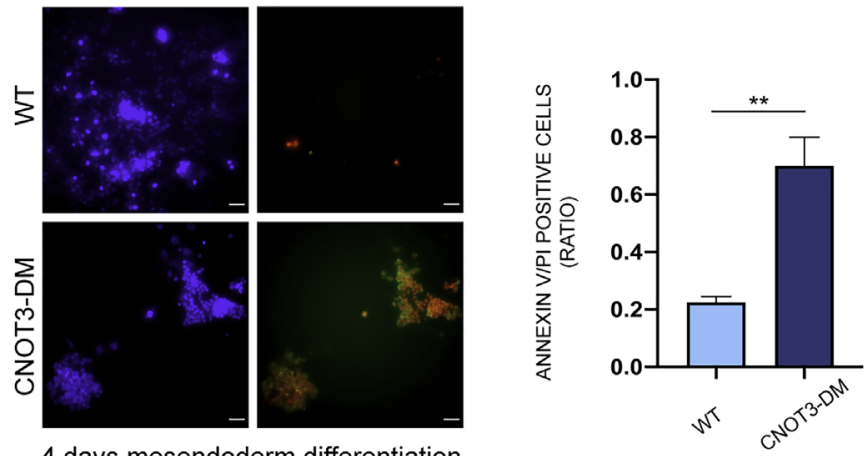

$\square$ WT

CNOT3-DM

4 days mesendoderm differentiation

FIGURE 4: Phosphorylation of CNOT3 prevents apoptosis of differentiating ME cells. (A) Top panel: Crystal violet staining of cells after $4 \mathrm{~d}$ BMP4 + FGF2-induced ME differentiation of WT and Cnot3-DM ESCs after 4 and $8 \mathrm{~d}$. Bottom panel: Live cells were counted based on trypan blue exclusion at the indicated time points. Histogram shows the percentage of live cells relative to the total number of live + dead cells at different time points of differentiation. For this panel and C, mean \pm SEM (unpaired $t$ test between WT and Cnot3-DM for each time point; $* \star P<0.01, \star \star \star P<0.001$; $n=3$ ). (B) Representative phase-contrast images showing the efficiency of BMP4 + FGF2-induced ME differentiation of WT and Cnot3-DM ESCs for 2, 4, and $8 \mathrm{~d}$. Scale bar: $100 \mu \mathrm{m}$. See also Supplemental Video 1 for a time-lapse analysis of the differentiations. (C) Immunofluorescence analysis of apoptotic markers Annexin V (green) and PI (red) in cells differentiated for $4 \mathrm{~d}$ with BMP4 + FGF2. Merged images show the Annexin V- and PI-stained apoptotic cells. Nuclei were stained with DAPI; scale bar $=100 \mu \mathrm{m}$. Histogram represents ratio of Annexin V/PI positive cells relative to total number of cells. Apoptotic cells were counted from 10 randomly chosen fields for each biological replicate.

Western blotting using an antibody that recognized the phosphorylated ERK TEY motif (Roux and Blenis, 2004) and an antibody against total ERK. The results showed a strong reduction in the level of phosphorylated ERK in the Cnot3-DM cells compared with wild type (Figure 5B). Levels of phosphorylated ERK and of total ERK1/2 were unaffected in undifferentiated Cnot3-DM ESCs (Supplemental Figure S7A). Aurora B and CNOT3 continued to interact in differentiating $\mathrm{ME}$ cells, as shown by immunoprecipitations carried out with anti-CNOT3 antibody on extracts from the 4-d differentiated wildtype and Cnot3-DM cells (Figure 5C). Levels of total CNOT3 were reduced in differentiated Cnot3-DM cells (Figure 5B), suggesting that phosphorylation by Aurora B stabilizes wild-type CNOT3.

\section{Altered interaction of mutant CNOT3 with Aurora B and ERK}

The proximity ligation assay (PLA) was used to analyze interactions between CNOT3, Aurora B, and ERK at the single-cell level. PLA analysis with mouse anti-Aurora $B$ and rabbit anti-CNOT3 antibod- ies after $4 d$ of differentiation of wild-type ESCs with BMP4 + FGF2 showed a high level of association between CNOT3 and Aurora B (Figure 6A). The number of interaction foci in the nuclei of wild-type cells was around half the number observed in the cytoplasm. Analysis of the Cnot3-DM cells showed a striking and unexpected increase of approximately threefold in the interaction between CNOT3 and Aurora B in the mutant cells compared with the wildtype cells and an increase in the proportion of interaction foci observed in the nucleus relative to the cytoplasm relative to wild-type cells (Figure 6A). Overall, these results confirm the strong interaction between CNOT3 and Aurora B that was observed by coimmunoprecipitation of extracts from undifferentiated ESCs and after $4 \mathrm{~d}$ of ME differentiation. The differences between the interaction patterns observed in the wild-type and Cnot3-T292A/S294A mutant cells raise the possibility of a dynamic cycle of phosphorylation of T292/S294 and subsequent release of Aurora B in differentiating wild-type ME cells, with the release blocked by the double mutation of the Aurora B target sites. 
A
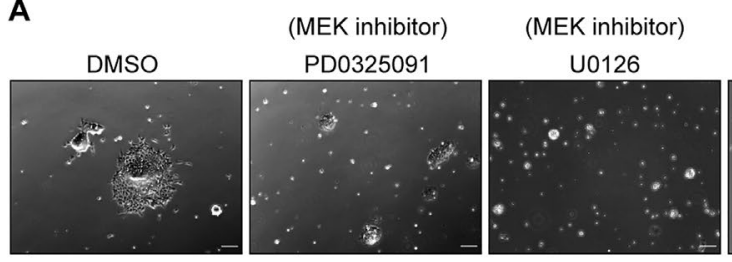

(FGFR inhibitor) AZD4547

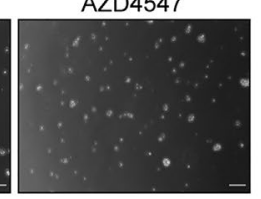

B

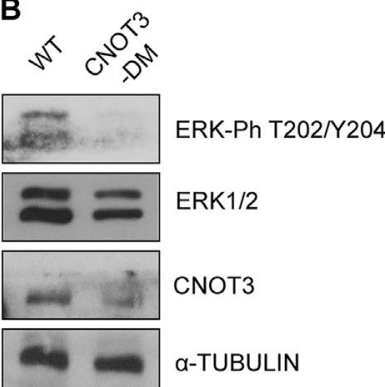

4-day mesendoderm

differentiated cells

FIGURE 5: The CNOT3 double mutation reduces ERK phosphorylation during ME

differentiation. (A) Phase-contrast images showing the effects of MEK inhibitors (PD0325091 and U0126), which abolish ERK activity, and the FGFR inhibitor AZD4547 on BMP4 + FGF2induced ME differentiation of wild-type (WT) ESCs. Differentiations were carried out for $4 \mathrm{~d}$. Each inhibitor was added after $2 \mathrm{~d}$ of differentiation. Vehicle $=$ DMSO. Scale bar $=100 \mu \mathrm{m}$.

(B) Representative immunoblot analysis of the indicated proteins carried out on cell extracts from WT and Cnot3-DM ESCs subjected to $4 \mathrm{~d}$ of BMP4 + FGF2-induced differentiation into ME. Loading control: $\alpha$-tubulin. (C) Coimmunoprecipitation was carried out with anti-CNOT3 antibody on cell extracts prepared from WT and Cnot3-DM ESCs differentiated as in B.

Coimmunoprecipitated proteins were immunoblotted and probed with anti-CNOT3 and antiAuorora B. Negative control: rabbit lgG. Input $=5 \%$ of extracts.

PLA of the interaction between CNOT3 and ERK showed a substantial level of interaction in wild-type 4-d differentiated cells that was broadly distributed between cytoplasmic and nuclear compartments (Figure 6B). The Cnot3-DM cells showed a threefold reduction in the number of interaction foci in the cytoplasm and a fivefold reduction in the nucleus. The strong effect of the double mutation on the interaction was confirmed with a second, separate antiCNOT3/anti-ERK antibody pair (Supplemental Figure S7B). The second antibody pair also showed a reduction in the numbers of foci in the cytoplasm and nucleus, but the nuclear to cytoplasmic ratios were similar in the wild-type and mutant cells, suggesting that the mutation affects cytoplasmic and nuclear interactions between CNOT3 and ERK to similar degrees.

Overall, the results described above provide compelling evidence that interaction of ERK with CNOT3 is promoted by Aurora B-mediated phosphorylation of CNOT3-T292/S294. The finding that mutation of the Aurora B phosphorylation sites reduced phosphorylation of ERK in ME cells (Figure 5B) suggests that the interaction between ERK and phosphorylated CNOT3 promotes ERK phosphorylation or stabilizes phosphorylated ERK, thereby enhancing Ras/MEK/ERK signaling. These observations provide evidence that the main effect of CNOT3 phosphorylation by Aurora B on MAPK/ERK signaling is to stabilize the phosphorylated form of ERK in the cytoplasmic and nuclear compartments, although a role for CNOT3 in transporting phospo-ERK into the nucleus cannot be completely ruled out.

In vitro pull down of ${ }^{35} \mathrm{~S}$-labeled CNOT3 with GST-ERK showed that ERK interacts directly with CNOT3 (Figure 6C). Analysis of CNOT3 deletion mutants showed some similarities to the binding of
4 days BMP4+FGF2

Aurora $B$, with deletion of the NOT box abolishing binding and deletion of the Cterminal 50 amino acids ( $\Delta 701-751)$ enhancing the interaction with GST-ERK. However, the effects of the CNOT3 deletions also showed some differences from the effects that we observed on Aurora B binding (Figure $1 \mathrm{C}$ ), with the $\Delta 651-700$ deletion mutant giving a $50 \%$ reduction in ERK binding, whereas the same deletion did not show a reduction in binding of Aurora B. This implies that there are differences in the contacts that Aurora B and ERK make with the CNOT3 NOT box region.

\section{Phosphorylation alters localization of CNOT3 in cancer cells}

Mutations in the coding region of human Cnot3 have been observed in a number of cancers (Forbes et al., 2017), with genetic analysis providing evidence that Cnot3 mutations can have tumor suppressor and tumor-promoting effects (De Keersmaecker et al., 2013; Delacruz et al., 2019). High levels of nuclear CNOT3 have also been observed in an aggressive colorectal cancer cell line (Cejas et al., 2017). To test whether Aurora B might be promoting nuclear localization and EMT/MET in cancer cells, H1299 and A549 non-small cell lung cancer (NSCLC) cells were stained with anti-CNOT3 antibody. $\mathrm{H} 1299$ was originally derived from a lymph node metastasis and A549 from a lung adenocarcinoma. The results of the immunofluorescence analysis (Figure 7A) showed that nuclear CNOT3 levels were approximately threefold higher than the cytoplasmic levels in $\mathrm{H} 1299$ cells, whereas the difference was 1.6-fold in A549 cells.

Involvement of Aurora B phosphorylation in the H1299-specific localization of CNOT3 was investigated by treating H1299 cells for $3 \mathrm{~h}$ with the Aurora B inhibitor AZD1152 (Figure 7B). The incubation time was optimized to avoid disruption of the cell cycle. The results showed a dramatic effect of Aurora B inhibition on CNOT3 localization in the H1299 cells with the nuclear levels of CNOT3 in the inhibitor-treated cells reduced by threefold relative to the cytoplasmic level (Figure 7B), confirming the involvement of Aurora B-mediated phosphorylation in driving nuclear localization of CNOT3.

The involvement of the NLS and adjacent Aurora B target sites in cancer is further supported by the recent identification of a Cnot3-K286E mutation in 11\% (4/37) of a cohort of premalignant adenomas that were sequenced from familial adenomatous polyposis (FAP) patients (Delacruz et al., 2019) (Figure 7C). Cnot3K286 is the first residue of the KKRGR NLS that is located adjacent to the sites of Aurora B phosphorylation in mouse ESCs. Mutations that affect the first residue of the Aurora B consensus (Cnot3-R290C and $-\mathrm{R} 290 \mathrm{H}$ ) have also been described in one lung and three colorectal cancers (Giannakis et al., 2016; Forbes et al., 2017; Priestley et al., 2019). Overall, mutations in the NLS have been observed in a total of nine patients with cancer or precancerous lesions (Figure 7C). This clustering of mutations provides strong evidence of a role for this region in oncogenic progression, particularly in colorectal cancers. 


\section{DISCUSSION}

The epithelial-to-mesenchymal transition (EMT) that leads to ME differentiation and gastrulation is a critical stage in mammalian embryogenesis and is also involved in metastasis of many human cancers. Our results identify a role for CNOT3 in regulating the signaling pathways that promote survival and proliferation of ME in the early embryo. The effect occurs through a direct interaction between CNOT3 and the Aurora B kinase, which is completely dependent on the NOT box domain of CNOT3 and results in Aurora B-mediated phosphorylation of specific sites adjacent to an NLS within the CNOT3 protein and increased localization of CNOT3 to the nucleus. The involvement of the NLS-adjacent Aurora B phosphorylation sites in the CNOT3 protein in germ layer formation was dramatically illustrated by the observation that mutation of the two Aurora B target residues strongly reduced the numbers of cells expressing mesoderm and endoderm markers in EBs formed from the mutant cells. In vitro differentiation of the mutant ESCs in a defined medium highlighted the importance of CNOT3 phosphorylation in promoting cell survival during differentiation of ME progenitor cells in response to FGF2 and BMP4.

Analysis of ERK phosphorylation showed that ERK activation is affected by the Cnot3 double mutation, with a strong reduction in the levels of phosphorylated ERK observed in the mutant ME cells. This finding provides a potential mechanistic explanation for the increased apoptosis observed in the Cnot3-DM cells when differentiation is induced using FGF2 and BMP4, a conclusion that is supported by the effect of MEK inhibitors on survival of differentiating ME cells. The fact that the double mutation had a particularly strong effect on survival of ME cells that were differentiated in response to the combination of Wnt, FGF2, and BMP4 raised the possibility that CNOT3 could also be involved in the cross-talk that is known to occur between the RAS/MEK/ERK and Wnt signaling pathways (Georgopoulos et al., 2014; Jeong et al., 2018).

Phosphorylation of ERK at the plasma membrane by MEK is followed by rapid import of phospho-ERK into the nucleus, which occurs primarily through interaction with the nuclear pore complex protein inportin-7 (James et al., 2007; Chuderland et al., 2008). In addition to being determined by the rate of shuttling into and out of the nucleus, the level of phospho-ERK in the nuclear compartment is known to be strongly dependent on binding of ERK to stabilizing proteins in the cytoplasm and in the nucleus (Formstecher et al., 2001; Mebratu and Tesfaigzi, 2009; Caunt and McArdle, 2012). The results of the in vitro GST pull down and in vivo PLA analyses indicate that ERK interacts with CNOT3 via the NOT box domain in differentiating ME cells and that this interaction is strongly reduced in both the cytoplasmic and nuclear compartments of the Cnot3-DM cells.

The observation that the double mutation increases interaction with Aurora B and reduces the interaction between CNOT3 and ERK suggests a possible model, which is illustrated schematically in Figure 8. According to this model, the two kinases would interact sequentially with CNOT3, with Aurora B first interacting with the CNOT3 NOT box, followed by phosphorylation of CNOT3 at the NLS-adjacent sites and release of the bound Aurora B, allowing ERK to bind to the NOT box. Our finding that Aurora B phosphorylation promotes localization of CNOT3 to the nucleus also suggests that the interaction between phosphorylated CNOT3 and ERK could be involved in stabilizing phospho-ERK in the cytoplasmic and nuclear compartments of normal somatic cells. This idea is supported by the finding that interactions between ERK and CNOT3 are reduced in both the nucleus and cytoplasm. However, we cannot rule out the possibility that other, as yet unidentified, functions of CNOT3 are involved in generating the phenotype of reduced survival during differentiation of the Cnot3-DM ME cells.

In addition to these findings, the increased nuclear localization of CNOT3 that we observe in an NSCLC cell line that was isolated from a lymph node metastasis opens up the possibility that Aurora B-dependent nuclear localization of CNOT3 affects signaling in cancer cells, acting as a driver for metastasis. The known association of elevated Aurora B levels with lymph node metastasis in a number of cancers (Tuncel et al., 2012; Takeshita et al., 2013; Zhang et al., 2015) and the well-established involvement of the MAPK/ERK and Wnt pathways in tumorigenesis and metastasis (Bang et al., 1998; Sebolt-Leopold et al., 1999; Smith et al., 2014; Stewart, 2014; Zhan et al., 2017) suggest that changes to the cytoplasmic-to-nuclear ratio of CNOT could be involved in progression of NSCLC.

A possible mechanistic rationale for the relocation of CNOT3 to the nuclei of metastatic $\mathrm{H} 1299$ cells comes from a proteomic analysis of phosphorylation targets that was used to compare kinase activities in A549 and H1299 cells (Sudhir et al., 2011). The results of this study revealed a dramatic difference between the two cell lines with the A549 adenoma cells showing a predominance of phosphorylated ERK targets, whereas phosphorylation in the H1299 cells derived from a lymph node metastasis was mediated mainly by Aurora B. Computer modeling has predicted that adaptor proteins will have concentrations that are optimal for activation of their cognate kinases, with an increase in the concentration of an adaptor above the optimal level for a particular compartment potentially reducing kinase activity (Levchenko et al., 2000; Witzel et al., 2012). This could explain the apparent parodox of very high levels of nuclear CNOT3 being associated with the reduced ERK activity that has been observed in $\mathrm{H} 1299$ cells. CNOT3 has also been shown to inhibit transcription of MHC Class II genes (Rodriguez-Gil et al., 2017), and $\mathrm{H} 1299$ cells do not express Class II genes, in contrast to A549 cells, which express them strongly (Neuwelt et al., 2020). Reduced Class II expression has been linked with lymph node metastasis (Warabi et al., 2000).

Overall, our results provide evidence of cross-talk between ERK and Aurora B in differentiating ME cells, with CNOT3 acting as an adaptor that links the two kinases. Our findings suggest that Aurora $B$ acts through CNOT3 to increase the level of phospho-ERK, thereby promoting survival of ME cells. These observations suggest that the synergy between the three proteins plays an important role in regulating cell survival during $\mathrm{ME}$ differentiation and gastrulation of the mouse embryo. The strong reduction in survival and proliferation that we observe when the Cnot3-DM cells are induced to differentiate into ME suggests that Aurora B links up with the MAPK/ ERK and Wnt pathways via CNOT3 to promote cell survival during the explosive expansion of cell numbers that occurs in the embryo at the time of implantation and gastrulation. Future experiments that examine the subcellular localization and intracellular dynamics of the interactions between CNOT3, Aurora B, and ERK should allow testing of the validity of the model shown in Figure 8. Additional insights should also come from analysis of interactions between CNOT3 and other components and regulators of the MAPK and Wnt signaling pathways.

\section{MATERIALS AND METHODS Cell culture}

E14 ESCs (female, 129/Ola) were obtained from the LMS Transgenics and ES cell facility. The ESCs were maintained on gelatin-coated plates, at $37^{\circ} \mathrm{C}, 5 \% \mathrm{CO}_{2}$, in KnockOut DMEM supplemented with $15 \%$ fetal bovine serum (FBS), 1× NEAA, 2 mM L-glutamine, $100 \mathrm{U} / \mathrm{ml}$ penicillin, $100 \mu \mathrm{g} / \mathrm{ml}$ streptomycin, amd $100 \mu \mathrm{M}$ 

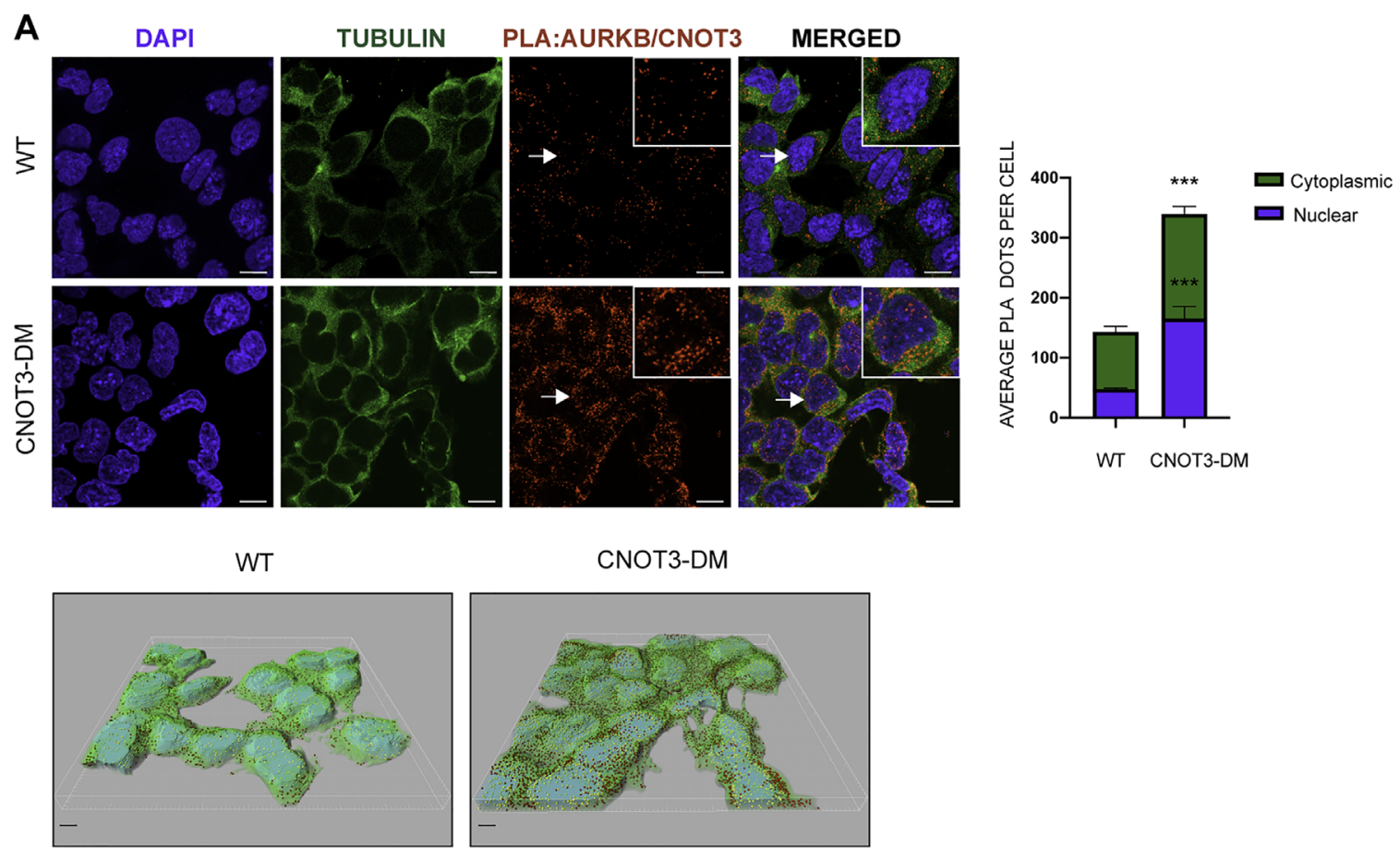

CNOT3-DM

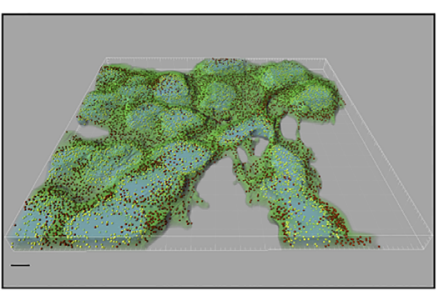

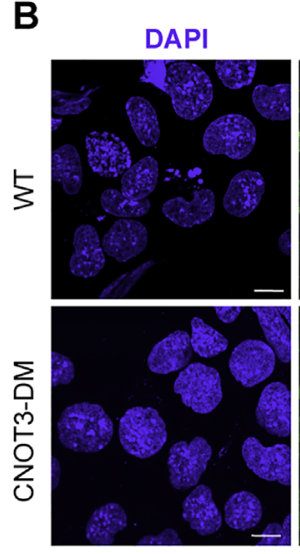

WT

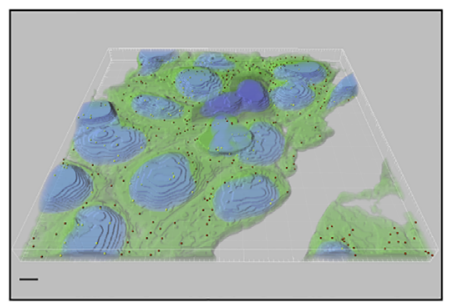

C
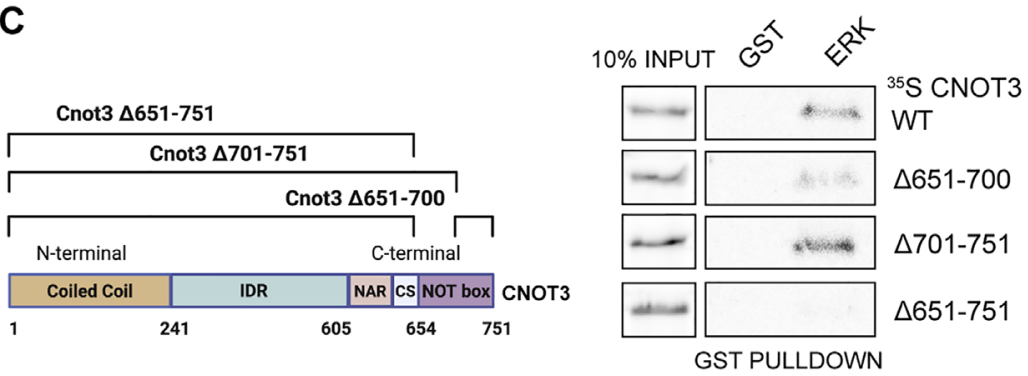

CNOT3-DM
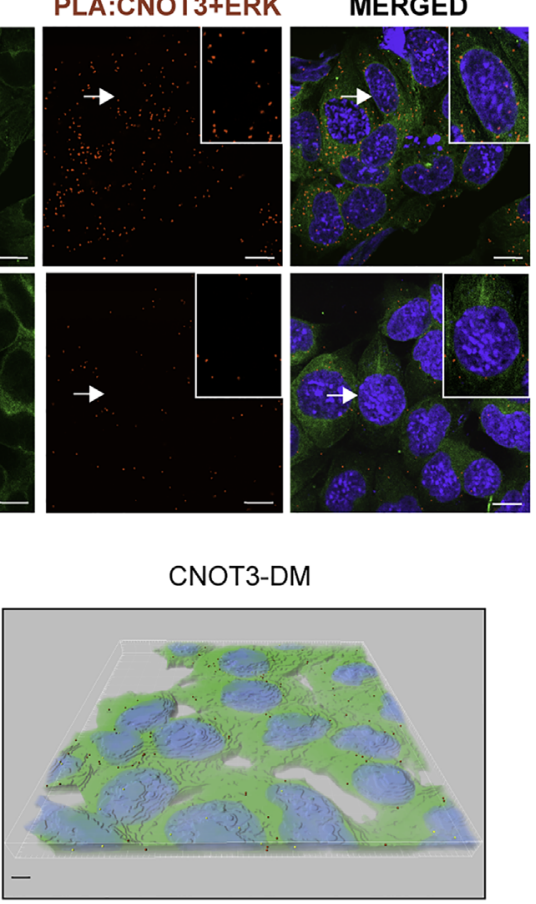

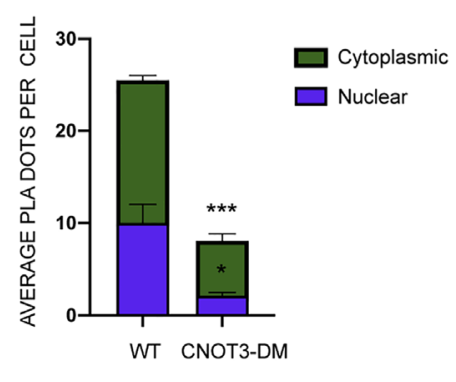

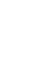

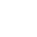


$\beta$-mercaptoethanol ( $\beta$-ME) (all reagents from Thermo Fisher Scientific, USA) and $1000 \mathrm{U} / \mathrm{ml}$ LIF (Merck, USA). HEK293 cells and A549 cells (American Type Culture Collection [ATCC]) were maintained in DMEM supplemented with 10\% FBS, 2 mM L-glutamine, $100 \mathrm{U} / \mathrm{ml}$ penicillin, and $100 \mu \mathrm{g} / \mathrm{ml}$ streptomycin. H1299 cells (ATCC, USA) were maintained in RPMI-1640 media supplemented with the same constituents as mentioned above.

\section{CNOT3 gene editing using CRISPR/Cas9}

The guide RNA (gRNA) used to generate the Cnot3-T292A/S294A mutant ESCs was 5'-GATTTAGACTTGGACCCACC-3'. The gRNA was cloned into pX330 (Addgene, USA; plasmid: 42230) using forward primer 5'-CACCGGATTTAGACTTGGACCCACC-3' and reverse primer 5'-AAACGGTGGGTCCAAGTCTAAATCC-3'

The 110-base-paired single-stranded (ss) donor DNA used to target Cnot3 in exon 10 carrying the mutations was $5^{\prime}$-ACTCTGAAGATGATAAGAAGAGAGGCCGATCTGCGGATGCTGAAGTCAGCCAGGTGGGTCCAAGTCTAAATCTGATGGTTTGTAACTTGTTTATTGCGTGGTCTCCAAAG-3'

Mouse ESCs $\left(4 \times 10^{6}\right.$ cells) were transfected with $3 \mu \mathrm{g}$ of $\mathrm{pX} 330$ plasmid carrying the gRNA, $4 \mu \mathrm{g}$ of the donor ssDNA, and $3 \mu \mathrm{g}$ of a puromycin resistance plasmid (pCAG-puro ${ }^{R}$ ) using the Mouse ES Cell Nucleofector Kit (Lonza, Switzerland) following the manufacturer's protocol. One day posttransfection, cells were subjected to puromycin selection $(1.5 \mu \mathrm{g} / \mathrm{ml})$ for $24 \mathrm{~h}$. A week after transfection, individual clones were picked and genotyped by allele-specific nested PCR. Mutant genotypes were confirmed by sequencing.

\section{Embryoid bodies}

Half a million cells (per well of a six-well plate) were seeded on ultralow-attachment plates (Corning Costar, USA) and maintained in KnockOut DMEM supplemented with 15\% FBS, 1× NEAA, 2 mM L-glutamine, $100 \mathrm{U} / \mathrm{ml}$ penicillin, $100 \mu \mathrm{g} / \mathrm{ml}$ streptomycin, and $100 \mu \mathrm{M} \beta-\mathrm{ME}$ and were grown up to $14 \mathrm{~d}$.

EBs were fixed in $4 \%$ paraformaldehyde for 20 min followed by permeabilization with $0.5 \%$ Triton-X for 20 min. Subsequently, EBs were blocked in $3 \%$ bovine serum albumin (BSA) for $1 \mathrm{~h}$ and then incubated with primary antibodies overnight at $4^{\circ} \mathrm{C}$. On the following day, the EBs were washed three times with phosphate-buffered saline (PBS) $+0.1 \%$ Tween-20 (PBST) and incubated for $1 \mathrm{~h}$ at room temperature with the appropriate secondary antibodies. EBs were then washed three times in PBST and incubated with $1 \mu \mathrm{g} / \mathrm{ml}$ 4',6-diamidino-2-phenylindole (DAPI) (Merck, USA) in PBS for
45 min. Images were acquired using a SP5 confocal microscope with LAS X software (Leica Microsystems, Germany) and were analyzed with Fiji ImageJ software (National Institutes of Health [NIH], USA).

\section{Differentiation experiments}

ESC differentiation into ME. Cells were plated at a density of 10,000 cells $/ \mathrm{cm}^{2}$ on gelatin-coated plates and incubated in DMEM/F12 KnockOut containing $64 \mu \mathrm{g} / \mathrm{ml}$ L-ascorbic acid-2phosphate magnesium, $543 \mu \mathrm{g} / \mathrm{ml}$ sodium bicarbonate, $1 \mu \mathrm{g} / \mathrm{ml}$ heparin, 1× insulin-transferrin-selenium, and $2 \mathrm{mM}$ glutamine. For the differentiations, different combinations of signaling factors were added to the medium (see Treatments of cells with ligands and inhibitors below). For time-lapse imaging of differentiation from day 3 to day 7 , the plate was transferred to an Axiovert 200 microscope (Zeiss, Germany) with environmental chamber (Solent Scientific Ltd., UK) and motorized stage (ASI, USA), and images were collected at an interval of $30 \mathrm{~min}$. Phasecontrast images were acquired in a DMIRE2 microscope (Leica Microsystems, Germany) using MetaMorph software.

ESC differentiation to endoderm. Cells were plated at a density of 10,000 cells $/ \mathrm{cm}^{2}$ on gelatin-coated plates and incubated in highglucose DMEM with $15 \%$ FBS, $100 \mathrm{U} / \mathrm{ml}$ penicillin, $100 \mu \mathrm{g} / \mathrm{ml}$ streptomycin, $0.1 \mathrm{mM}$ nonessential amino acids, $1 \mathrm{mM} \mathrm{MTG}, 1 \times$ GlutaMAX and supplemented with $25 \mathrm{ng} / \mathrm{ml} \mathrm{FGF2} \mathrm{(Merck,} \mathrm{USA)} \mathrm{and} 10$ $\mu \mathrm{M}$ retinoic acid (Merck, USA) for $3 \mathrm{~d}$ (Kim et al., 2010a).

ESC differentiation to mesoderm. Cells were plated at a density of 15,000 cells $/ \mathrm{cm}^{2}$ in gelatin + fibronectin-coated plates and incubated for $4 \mathrm{~d}$ in DMEM/F12-Neurobasal (1:1), N2, B27, 1× GlutaMAX, $100 \mathrm{U} / \mathrm{ml}$ penicillin, $100 \mu \mathrm{g} / \mathrm{ml}$ streptomycin, $0.1 \% \beta-\mathrm{ME}$ and $30 \mathrm{ng} / \mathrm{ml}$ activin A (R\&D Systems, USA).

ESC differentiation to ectoderm. Cells were plated at a density of $15,000 \mathrm{cells} / \mathrm{cm}^{2}$ on gelatin-coated plates and incubated for $4 \mathrm{~d}$ in DMEM/F12-Neurobasal (1:1), 1× GlutaMAX, $100 \mathrm{U} / \mathrm{ml}$ penicillin, $100 \mu \mathrm{g} / \mathrm{ml}$ streptomycin, $100 \mu \mathrm{M} \beta-\mathrm{ME}$, B27 minus vitamin $\mathrm{A}$ and N2.

\section{Treatment of cells with ligands and inhibitors}

Aurora B inhibitor AZD1152 (Merck, USA), 200 nM; human recombinant Wnt3 (Cloud-Clone Corp, USA), $200 \mathrm{ng} / \mathrm{ml}$; activin A (R\&D Systems, USA), $100 \mathrm{ng} / \mathrm{ml}$; BMP4 (Merck, USA), $10 \mathrm{ng} / \mathrm{ml}$; FGF2

FIGURE 6: Phosphorylation of CNOT3 alters its interaction with Aurora B and ERK in ME cells. (A, B) PLA was used to detect interaction between endogenous Aurora B (AURKB) and CNOT3 (A) and CNOT3 and ERK (B) after $4 d$ of BMP4 + FGF2-induced differentiation of ME cells from WT and Cnot3-DM ESCs. Top panels: Red dots represent positive PLA signals. Nuclei were stained with DAPI and cytoplasm with anti-tubulin. Boxed areas show an enlarged image of a cell (indicated by the arrows) from each merged panel. Scale bars $=10 \mu \mathrm{m}$. Bottom panels: Three-dimensional digital reconstructions showing the distribution of PLA dots in the nucleus (yellow) and cytoplasm (red). Scale bars $=5 \mu \mathrm{m}$. PLA dots were quantified from randomly chosen fields from at least 50 cells for each biological replicate. Histograms represent average number of interactions per cell (dots/cell) in the nucleus (blue) and cytoplasm (green). Mean \pm SEM (unpaired $t$ test; ${ }^{\star \star \star} P<0.001 ;(A) n=3$, (B) $n=2$. Single antibody controls and PLA of CNOT3 and ERK using a second set of antibodies are shown in Supplemental Figure S7, B and C. (C) In vitro GST pull-down assay using GST-ERK1 and in vitro transcribed/translated wild-type (WT) CNOT3 and CNOT3 deletion mutants labeled with ${ }^{35} \mathrm{~S}$-methionine. Left panel: Schematic representation of the Cnot3 deletion mutants. Middle panel: Representative in vitro GST pull-down assay of GST-ERK1 and ${ }^{35}$ S-labeled WT CNOT3 and CNOT3 deletion mutants. GST-only was used as a negative control for each pull down. Input $=10 \%$ of the respective labeled proteins. Right panel: Histogram shows quantification of the band intensities normalized to input and relative to WT CNOT3. Mean $\pm \operatorname{SEM}(n=3)$ (unpaired $t$ test; $* P<0.05$,

$\star \star P<0.01, * \star \star P<0.001)$ 
A
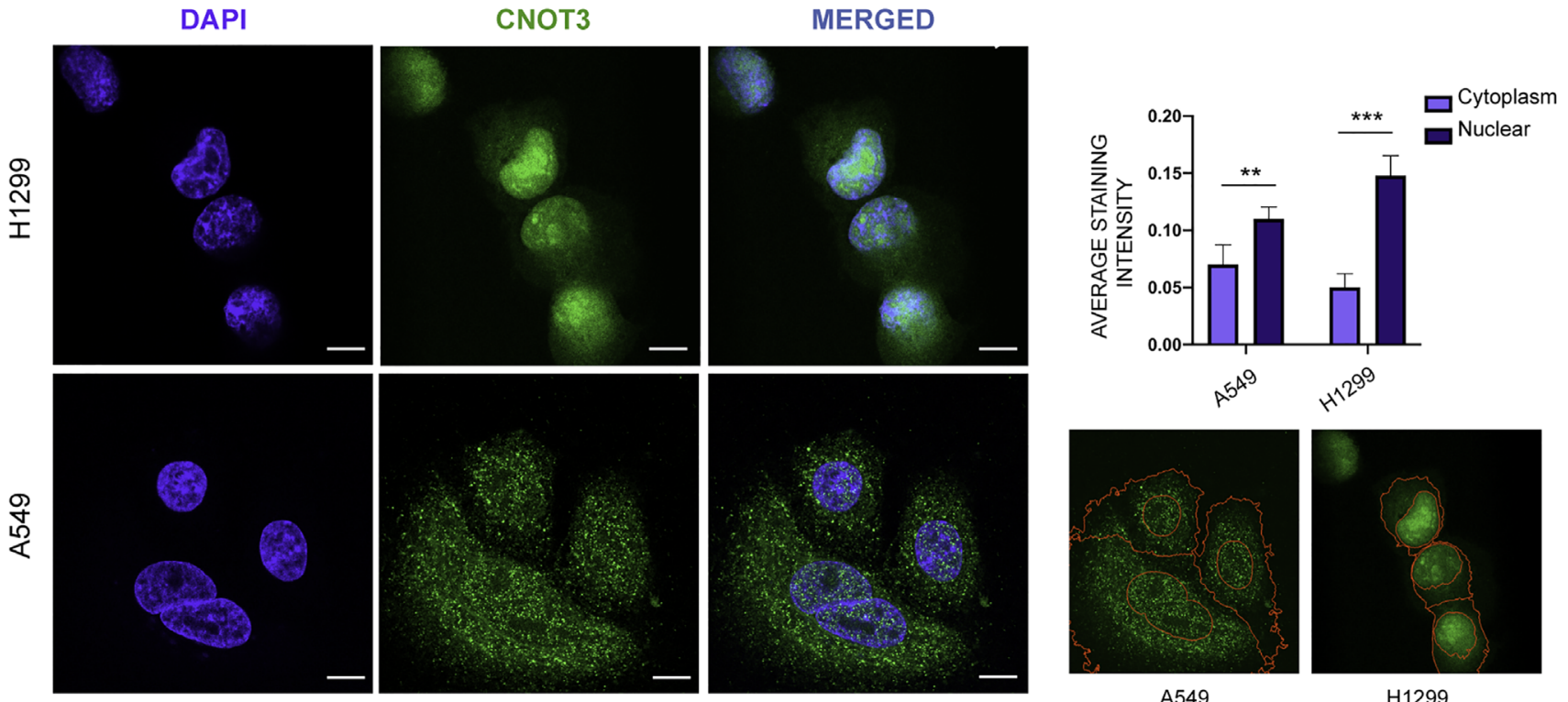

B
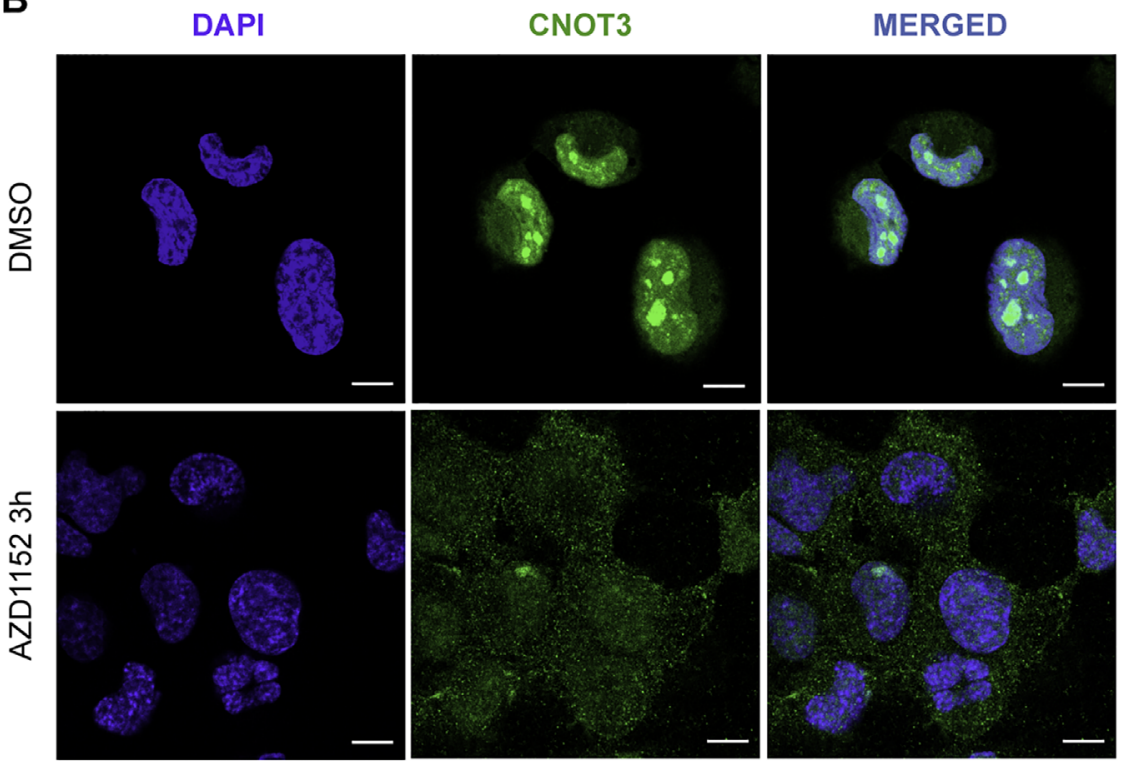

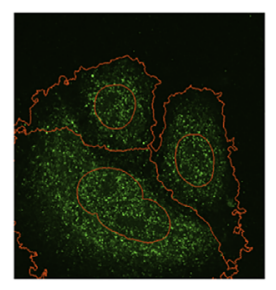

A549

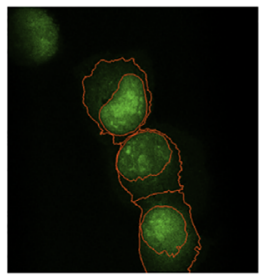

H1299
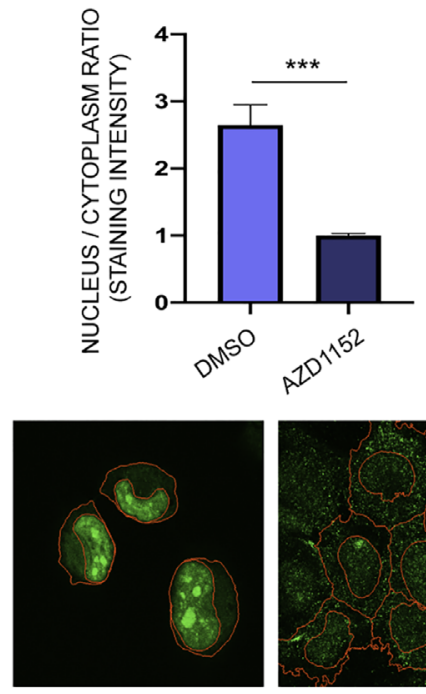

DMSO

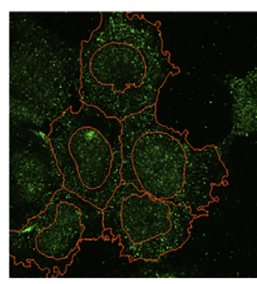

AZD1152

H1299 cells

C

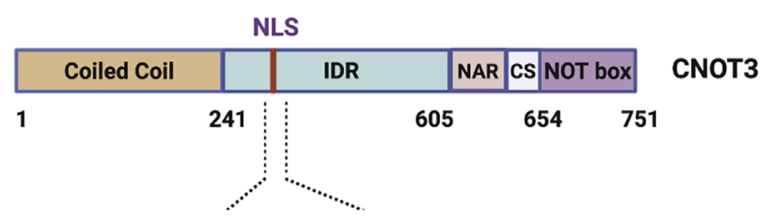

PTSTTSSSPIPPSPANCTTENSEDDKKRGRSTDSEVSQSPAKNGSKPVHSNQHPQSPAVP...

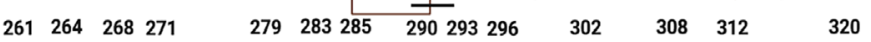

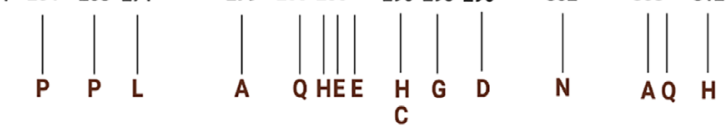

\begin{tabular}{|ccc|}
\hline Mutation & $\begin{array}{c}\text { Number of } \\
\text { patients }\end{array}$ & Tissue \\
\hline T264P & 1 & Thyroid \\
S268P & 1 & Upper aerodigestive tract \\
P271L & 1 & Lung \\
T279A & 1 & Ovary \\
E283Q & 1 & Urinary tract \\
D285H & 1 & Breast \\
\hline K286E & 4 & Colon \\
K287E & 1 & Colon \\
R290C & 2 & Colon \\
R290H & 2 & Lung, Colorectal \\
\hline D293G & 1 & Lung \\
V296D & 1 & Colon \\
K302N & 1 & Endometrium \\
V308A & 1 & Liver \\
H309Q & 1 & Liver \\
Q312H & 2 & Liver \\
\hline
\end{tabular}

mutations observed in multiple patients 
(Merck, USA), 25 ng/ml; CHIR99021(Merck, USA), 3 нM; PD0325901 (Merck, USA), 500 nM; U0126 (Merck, USA), 10 M; AZD4547 (Abcam, UK), $5 \mathrm{nM}$.

\section{Cell survival assay and Annexin $\mathrm{V}$ staining}

The Annexin $V$ staining was performed using the FITC Annexin $V$ apoptosis detection kit with PI (BioLegend, USA). The cells were washed with PBS followed by washing with staining buffer and resuspension in binding buffer containing anti-Annexin $\mathrm{V}$ and $\mathrm{PI}$ and incubation for $15 \mathrm{mins}$ at room temperature in the dark. The cells were finally washed with binding buffer and incubated with $1 \mu \mathrm{g} / \mathrm{ml}$ DAPI in PBS for 5 min. Images were acquired using an IX70 microscope (Olympus, Japan) with Micro-manager software.

Cells were stained with $0.2 \%$ crystal violet for a gross estimation of efficiency of differentiation. At different time points of differentiation, live and dead cells were distinguished by trypan blue staining and counted manually. Cell survival was measured using Cell Proliferation Reagent WST-1 (Merck, USA) at a final dilution of 1:10 followed by incubation for $0.5 \mathrm{~h}$ and quantitation with a scanning multiwell spectrophotometer (SpectraMax-Molecular Devices, USA).

\section{Immunocytochemistry}

All differentiated cells were grown in gelatin coated $\mu$-slides (ibidi, Germany) for immunofluorescence. Cells were fixed in $4 \%$ paraformaldehyde for $10 \mathrm{~min}$, permeabilized with $0.5 \%$ Triton-X for $15 \mathrm{~min}$, and blocked in 3\% BSA for $1 \mathrm{~h}$. Following incubation with primary antibodies at $4^{\circ} \mathrm{C}$ overnight, the cells were washed with PBST and incubated for $1 \mathrm{~h}$ at room temperature with the appropriate secondary antibodies. DAPI was used to stain the nuclei. Images were acquired using a SP5 confocal microscope with LAS X software (Leica Microsystems, Germany) or IX70 microscope with Micro-manager software. Images were analyzed with Fiji ImageJ software $(\mathrm{NIH}$, USA). Quantitative analysis of staining of ME cells and H1299 and A549 cancer cells was carried out using CellProfiler software (Broad Institute, USA). The antibodies used are listed in Supplemental Table S2.

\section{Expression plasmids}

Full-length Cnot3 cDNA (OriGene, USA) was cloned in pCDNA 3.2/ V5/GW/D-TOPO by PCR addition of restriction sites Smal/Notl following the manufacturer's instructions. Single Cnot3-T292A, Cnot3S294A mutant constructs and the T292A/S294A double mutant were generated by site directed mutagenesis and cloned as above. An $8 \mu \mathrm{g}$ aliquot of each DNA construct was transfected into HEK293 cells by the calcium phosphate method. Deletion fragments Cnot3
$\Delta$ 1-200, Cnot3 $\Delta 651-700$, Cnot3 $\Delta 701-751$ and Cnot3 $\Delta 651-751$ were synthesized (Genewiz, UK) and cloned in pCDNA 3.2/N5/GW/ D-TOPO using restriction sites Kpnl/Ascl. pCDNA3-T7-ERK1 (Addgene, USA; plasmid:14440) and full-length Aurora B cDNA (Dharmacon-Horizon Discovery, UK) was cloned in pGEX-4T1.

\section{Immunoprecipitation}

Cells were harvested in immunoprecipitation lysis buffer $(50 \mathrm{mM}$ Tris$\mathrm{HCl}, \mathrm{pH} 7.4,150 \mathrm{mM} \mathrm{NaCl}, 10 \%$ glycerol, 1\% Nonidet P-40, $1.0 \mathrm{mM}$ EDTA) with Complete protease inhibitor cocktail (Merck, USA). In all cases, $500 \mu \mathrm{g}$ of total protein was used. Extracted proteins were immunoprecipitated with Protein A Sepharose CL-4B beads (GE Healthcare, USA) and the desired primary antibodies. The immunocomplexes were eluted by boiling with $2 \times$ SDS loading buffer.

\section{Protein extractions from cells}

To obtain the whole cell extracts, cells were washed with ice-cold PBS and the cell pellet was reuspended in Tris lysis buffer $(50 \mathrm{mM}$ Tris- $\mathrm{HCl}, \mathrm{pH} 7.5,150 \mathrm{mM} \mathrm{NaCl}, 1 \%$ Triton X-100, 1 mM dithiothreitol [DTT], $1 \mathrm{mM} \mathrm{Na}_{3} \mathrm{VO}_{4}$ ) with Complete protease inhibitor cocktail (Merck, USA).

For preparation of cytoplasmic and nuclear extracts, cells were harvested and resuspended in harvest buffer containing $10 \mathrm{mM}$ HEPES (pH 7.9), $50 \mathrm{mM} \mathrm{NaCl}, 0.5 \mathrm{M}$ sucrose, $0.1 \mathrm{mM}$ EDTA, $0.5 \%$ Triton X-100 and with Complete protease inhibitor cocktail (Merck). After obtaining the cytoplasmic extract, the nuclear pellet was further washed with wash buffer/buffer A (10 mM HEPES [pH 7.9], $10 \mathrm{mM} \mathrm{KCl}, 0.1 \mathrm{mM}$ EDTA, and $0.1 \mathrm{mM}$ EGTA) and then resuspended in buffer C (10 mM HEPES [pH 7.9], $500 \mathrm{mM} \mathrm{NaCl}, 0.1 \mathrm{mM}$ EDTA, $0.1 \mathrm{mM}$ egtazic acid [EGTA], 0.1\% NP40) and protease inhibitor cocktail to extract the nuclear proteins.

\section{Immunoblotting}

Immunoprecipitated proteins in loading buffer or equal amounts of proteins obtained from cell extracts (diluted in 5x SDS loading buffer) were boiled for $5 \mathrm{~min}$ and subjected to SDS-PAGE. Gels were transferred to nitrocellulose membranes (GE Healthcare, USA). Membranes were blocked with $5 \%$ BSA or $5 \%$ milk for $1 \mathrm{~h}$ at room temperature and incubated with the desired primary antibodies overnight at $4^{\circ} \mathrm{C}$. On the following day, membranes were washed three times with Tris-buffered saline-Tween 20, incubated with the appropriate secondary antibodies (dilution 1:10,000) for $1 \mathrm{~h}$ at room temperature, washed, and developed using Crescendo ECL (Merck, USA) using x-ray films on a Photon Imaging System (UK) or Amersham Imager 680 (GE Healthcare, USA).

FIGURE 7: Phosphorylation by Aurora B promotes nuclear localization of CNOT3 in a metastatic NSCLC cell line. (A) Comparison of nuclear and cytoplasmic localization of CNOT3 in two NSCLC cell lines. The H1299 line was derived from a lymph node metastasis and the A549 line from a lung adenocarcinoma. Left panel: H1299 cells (top) and A549 cells (bottom) immunostained with anti-CNOT3 and counterstained with DAPI (scale bar: $10 \mu \mathrm{m}$ ). Right panel (top): Histogram shows digital quantification of the average intensities of the nuclear and cytoplasmic staining for CNOT3 in the $\mathrm{H} 1299$ and A549 cells. For this panel and B, mean \pm SEM; $n=3$ (unpaired $t$ test; ${ }^{\star \star} P<0.01, \star \star \star P<0.001$ ). Right panel (bottom) shows representative fields depicting the segmentation of cells and nuclei that was used for quantification. This was performed on at least 30 cells from randomly chosen fields for each biological replicate. (B) Effect of the Aurora B inhibitor AZD1152 on nuclear and cytoplasmic localization of CNOT3 in H1299 cells. Left panel: $\mathrm{H} 1299$ cells were incubated for $3 \mathrm{~h}$ with AZD1152 (bottom) or vehicle (DMSO, top) and were then immunostained as in A (scale bar $=10 \mu \mathrm{m}$ ). Right panel: Histogram shows quantification of the average ratio of nuclear to cytoplasmic staining for CNOT3 in the AZD1152- and vehicle-treated cells. Digital quantification as in A. (C) Missense mutations described in human cancer patients in the region of CNOT3 spanning residues 261-320. Blue spheres indicate mutations that have been observed in more than one patient. The NLS is indicated by rectangles in the left and right panels, and the Aurora $B$ consensus is underlined in the left panel. $\mathrm{P}=$ phosphorylation sites. 


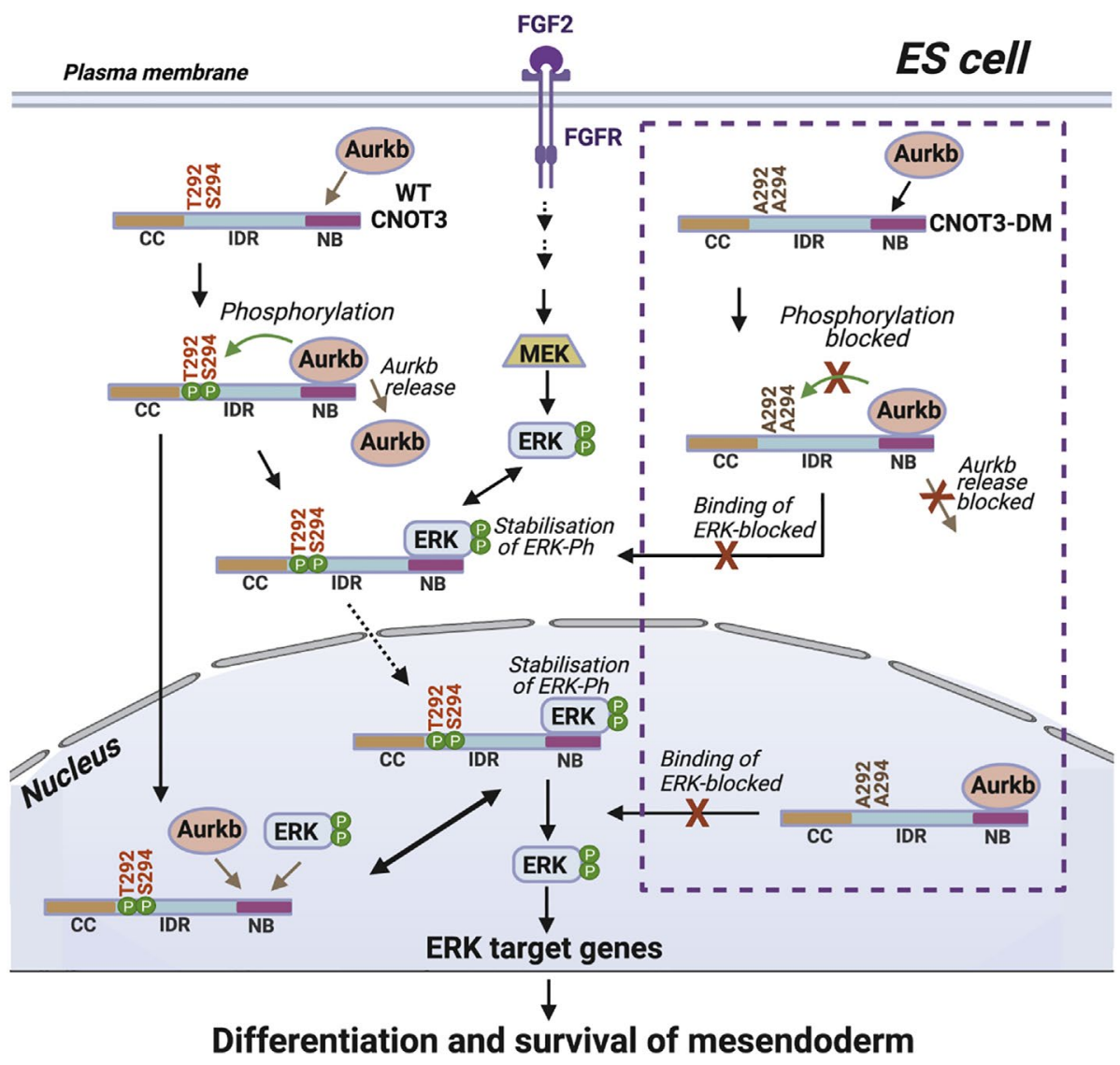

FIGURE 8: Schematic model showing the proposed regulation of ERK activity by Aurora B and CNOT3. Aurora B (Aurkb) binds to the NOT box (NB) of CNOT3 (for clarity the additional synergistic contact with the amino-terminal coiled-coil [CC] domain is not shown). Our results indicate that bound Aurora B phosphorylates CNOT3-T292/S294, located adjacent to a NLS in the intrinsically disordered region (IDR) of CNOT3 and promotes uptake of CNOT3 into the nucleus. The model proposes that phosphorylation of CNOT3 also causes bound Aurora B to be released, allowing phospho-ERK (ERK-Ph) to bind to the NOT box. This would result in stabilization of ERK-Ph in the cytoplasm and nucleus and might also facilitate transport of ERK-Ph into the nucleus. Mutation of CNOT3-T292/S294 to A (CNOT3-DM) prevents phosphorylation of these residues by Aurora B (depicted inside the dashed box). This would block release of Aurora B and prevents binding of ERK to the NOT box, resulting in destabilization and down-regulation of ERK-Ph. Created with BioRender.com.

GST pull-down assay

To perform the GST pull down with GSTAurora B and GST-ERK1 with Cnot3 and the deletion mutants of $\mathrm{Cnot} 3,0.5 \mu \mathrm{g}$ of pCDNA-Cnot3, pCDNA-Cnot3 $\triangle 1-200$, pCDNA-Cnot3 $\triangle 651-700$, pCDNA-Cnot3 $\triangle 701-751$, and pCDNA-Cnot3 $\Delta 651-751$ were in vitro transcribed/translated using a TNT Quick Coupled Transcription/Translation kit (Promega, USA) according to the manufacturer's instructions using $10 \mu \mathrm{Ci}$ of ${ }^{35} \mathrm{~S}$-methionine to radiolabel the proteins. GST or GST-Aurora B or GST-ERK $(1 \mu \mathrm{g})$ was added to the GSH beads (GE Healthcare, USA) in binding buffer (50 mM Tris$\mathrm{Cl}, \mathrm{pH}$ 8.0, $150 \mathrm{mM}$ monopotassium glutamate, $1 \mathrm{mM}$ EDTA, 0.1\% Igepal CAL630, $5 \%$ glycerol, $0.2 \%$ BSA), supplemented with Complete protease inhibitor cocktail (Merck, USA), and incubated for $2 \mathrm{~h}$ at $4{ }^{\circ} \mathrm{C}$. The beads were then washed and 5 $\mu \mathrm{l}$ of the in vitro transcribed/translated CNOT3 was incubated with the beads overnight at $4^{\circ} \mathrm{C}$. The beads were then washed with the binding buffer, and the proteins were eluted by boiling in loading buffer. The eluted proteins were subjected to SDS-PAGE, stained, dried for $1 \mathrm{~h}$ at $80^{\circ} \mathrm{C}$, and exposed overnight to Phosphor screen in a cassette (GE Healthcare, USA). Images were captured in a Fujifilm FLA 5100 scanner (Japan) using Fujifilm FLA5000 software.

\section{In vitro kinase assay}

pCDNA-Cnot3-T292A, pCDNA-Cnot3S294A, pCDNA-T292A/S294A, and pCDNA-Cnot3 $\Delta 651-751$ constructs were in vitro transcribed/translated as described above, and the resultant V5tagged proteins were purified using a V5tagged protein purification kit ver.2 (MBL International, USA). Purified proteins were

\section{Protein purification from bacteria}

PGEX-4T1-Aurora B and pGEX-4T1-ERK1 were transformed into competent BL21 Escherichia coli cells. To induce protein expression, $\beta$-D-1 thiogalactopyranoside (IPTG) (Merck, USA) was added at a concentration of $0.5 \mathrm{mM}$ and the cells were grown for $3 \mathrm{~h}$ at $37^{\circ} \mathrm{C}$. The cell pellets were resuspended in PBS $+0.5 \%$ Triton X-100 supplemented with Complete protease inhibitor cocktail (Merck, USA) and frozen overnight at $-80^{\circ} \mathrm{C}$ for lysis. The following day, the mix was incubated with lysozome $(1 \mathrm{mg} / \mathrm{ml})$ followed by sonication at intervals of $15 \mathrm{~s}$ on and $45 \mathrm{~s}$ off for $5 \mathrm{~min}$. The clear supernatants were collected, and Sepharose High performance (GSH) beads (GE Healthcare, USA) were added followed by overnight incubation at $4^{\circ} \mathrm{C}$. Beads were then washed with PBS $+0.5 \%$ Triton X-100, and the GST fusion proteins were eluted with elution buffer $(10 \mathrm{mM}$ glutathione reduced, $5 \%$ glycerol in $50 \mathrm{mM}$ Tris- $\mathrm{Cl}$, $\mathrm{pH}$ 8.0) for $15 \mathrm{~min}$ at room temperature followed by dialysis in 50 $\mathrm{mM}$ Tris-Cl, pH 8.0, $0.15 \mathrm{M} \mathrm{NaCl}, 10 \%$ glycerol, $0.5 \mathrm{mM} \mathrm{DTT}$, and $0.5 \mathrm{mM}$ phenylmethylsulfonyl fluoride (PMSF). used as substrate and incubated with $80 \mathrm{ng}$ of GST-Aurora B (ProQuinase, Germany) for $30 \mathrm{~min}$ at $30^{\circ} \mathrm{C}$ in phosphorylation buffer (50 mM Tris- $\mathrm{HCl}, \mathrm{pH} 7.5,10 \mathrm{mM} \mathrm{MgCl}_{2}, 500 \mu \mathrm{M}$ ATP, 1 mM DTT, $5 \mathrm{mM} \mathrm{NaF}, 1 \mu \mathrm{l}$ of ${ }^{32} \mathrm{P} \gamma$-ATP $\left.10 \mu \mathrm{Ci}\right)$. Reactions were stopped by the addition of SDS loading buffer, boiled for $5 \mathrm{~min}$, and subsequently run on SDS-PAGE. The gel was dried and exposed to $x-$ ray film.

\section{Flow cytometry}

Cells were trypsinised, washed twice with PBS, fixed with 70\% ethanol for 30 min on ice, and washed twice with $2 \%$ FCS-PBS. Subsequently, cells were resuspended in PBS containing $1 \mu \mathrm{g} / \mathrm{ml}$ RNase A (Thermo Fisher Scientific, USA), $50 \mu \mathrm{g} / \mathrm{ml}$ propidium iodide, and $0.05 \% \mathrm{NP} 40$, and incubated $20 \mathrm{~min}$ at room temperature in the dark followed by analysis. Anaysis was performed on an LSRII Flow Cytometer (Becton-Dickinson, USA), and data were analyzed using FlowJo Software (BectonDickinson, USA). 


\section{RNA sequencing}

Total RNA was extracted from $2 \times 10^{6}$ cells (three biological replicates for wild-type and CNOT3-DM cells) using Trizol reagent (Thermo Fisher Scientific, USA) following the manufacturer's instructions. A $2 \mu \mathrm{l}$ aliquot of a 1:10 dilution of the ERCC RNA Spikein Mix (Thermo Fisher Scientific) were added to each sample. The quality of the extracted RNA was analyzed using the RNA 6000 Nano kit on a 2100 Bioanalyzer (Agilent, USA). An aliquot of $500 \mathrm{ng}$ of RNA was used to prepare a polyadenylated RNA library with the TruSeq Stranded mRNA Library Prep Kit (Illumina, USA) following the manufacturer's protocol. RNA libraries were sequenced in one lane by multiplexing on an Illumina HiSeq 2500 sequencer with a 100 base pair read output. RNAseq reads were aligned to Ensembl Mouse genome (NCBIM37) reference sequence assembly and transcript annotation that was obtained from Illumina iGenomes (https://support.illumina.com/sequencing/sequencing_software/ igenome.html) and to ERCC reference with Tophat2 (2.0.11) (Kim et al., 2013). Gene-based read counts and ERCC counts were obtained using the featureCounts function from the Rsubread Bioconductor package (Liao et al., 2013, 2014). Differentially expressed gene analysis was performed with the DESeq2 Bioconductor package (Love et al., 2014) after normalizing the data against ERCC with the RUVseq Bioconductor package (Risso et al., 2014). Differentially expressed genes were defined with the Benjamini-Hochberg adjusted $p$ value $<0.05$ and fold change ratio $>1.5$. Gene ontology analysis was performed with the goseq Bioconductor package (Young et al., 2010). After converting the mouse gene symbol to a human gene symbol using the report of Human and Mouse Homology retrieved from Mouse Genome Informatics (MGl; www.informatics.jax.org), Gene Set Enrichment Analysis (GSEA) (Mootha et al., 2003; Subramanian et al., 2005) was performed with the GseaPreranked tool using the Hallmarks gene set (h.all.v5.2.symbols.gmt).

\section{Proximity ligation assay}

The PLA was performed using the Duolink In Situ Red Starter Kit Mouse/Rabbit (Merck, USA) following the manufacturer's instructions. Images were acquired using a SP5 confocal microscope with LAS X software (Leica Microsystems, Germany). PLA dots were analyzed and quantified using Imaris Bitplane software (Oxford Instruments, UK). Three-dimensional segmentation and digital image reconstructions of the cells were carried out using the Imaris Spots and Surfaces packages.

\section{Mass spectrometry}

Peptides were separated using an Ultimate 3000 RSLC nano liquid chromatography system (Thermo Fisher Scientific, USA) coupled to an LTQ Velos Orbitrap mass spectrometer (Thermo Fisher Scientific, USA) via a Dionex nano-esi source. Eluted peptides were analyzed by the LTQ Velos operating in positive polarity using a data-dependent acquisition mode. Ions for fragmentation were determined from an initial MS1 survey scan at 60,000 resolution (at $\mathrm{m} / \mathrm{z} 200$ ). Ion Trap CID (collisional induced dissociation), Ion Trap CID-MSA (Multi Stage Activation), and HCD (higher energy collisional induced dissociation) were carried out concurrently on the top three most abundant ions. For CID fragmentation methods MS1 and MS2 MSn AGC targets were set to $1 \mathrm{e} 6$ and $1 \mathrm{e} 4$ for one microscan and a maximum injection time of 500 and $100 \mathrm{~ms}$, respectively. A survey scan m/z range of 350-1500 was used, with a normalized collision energy set to $35 \%$ for both CID and HCD, charge state rejection enabled for +1 ions, and a minimum threshold for triggering fragmentation of 500 counts.

\section{RNA isolation and qPCR}

Total RNA was isolated with TRIzol reagent (Thermo Fisher Scientific, USA) following the manufacturer's instructions. cDNA was synthesized with the RevertAid First Strand cDNA Synthesis Kit (Thermo Fisher Scientific, USA) using 200 ng of RNA following the manufacturer's protocol. qPCRs were performed using Sensimix SYBR NORox SYBR GREEN (Bioline, UK). Each PCR was performed in duplicate using $1 \mu \mathrm{l}$ of cDNA (from a $20 \mu \mathrm{l}$ reaction) and $200 \mathrm{nM}$ primer concentration. Gene expression was determined relative to MLN 51 transcript levels. The primers used in this analysis are listed in Supplemental Table S1.

\section{Statistical analysis}

All statistical analyses were performed with GraphPad Prism software (GraphPad, USA). The statistical tests used in each experiment and significances are indicated in the corresponding figure legends. For all experiments involving cells, the value $n$ refers to the number of biological replicates of the experiments that were carried out. For the in vitro pull-down experiments, $n$ refers to the number of separate experiments used to generate the values shown in the histograms.

\section{DATA AVAILABILITY}

The RNA-seq data have been deposited at GEO under accession number GSE138213.

\section{ACKNOWLEDGMENTS}

We thank Nicola Festuccia, Aida di Gregorio, and Sheila Xie for advice and helpful discussions; Holger Kramer and Alex Montoya for assistance with mass spectrometry data analysis; and Lepakshi Ranjha for advice on the ${ }^{35} \mathrm{~S}$ pull-down and in vitro kinase assays. We also thank the staff of the LMS Genomics Facility for HT sequencing, and the London Institute of Medical Sciences/National Institute for Health Research, Imperial Biomedical Research Centre Flow Cytometry Facility for support. This research was funded by the Medical Research Council UK.

\section{REFERENCES}

Alexanian M, Maric D, Jenkinson SP, Mina M, Friedman CE, Ting C-C, Micheletti R, Plaisance I, Nemir M, Maison D, et al. (2017). A transcribed enhancer dictates mesendoderm specification in pluripotency. Nat Commun 8, 1806.

Bakre MM, Hoi A, Mong JC, Koh YY, Wong KY, Stanton LW (2007). Generation of multipotential mesendodermal progenitors from mouse embryonic stem cells via sustained Wnt pathway activation. J Biol Chem 282, 31703-31712.

Bang YJ, Kwon JH, Kang SH, Kim JW, Yang YC (1998). Increased MAPK activity and MKP-1 overexpression in human gastric adenocarcinoma. Biochem Biophys Res Commun 250, 43-47

Ben-Haim N, Lu C, Guzman-Ayala M, Pescatore L, Mesnard D, Bischofberger M, Naef F, Robertson EJ, Constam DB (2006). The nodal precursor acting via activin receptors induces mesoderm by maintaining a source of its convertases and BMP4. Dev Cell 11, 313-323.

Bernardo AS, Faial T, Gardner L, Niakan KK, Ortmann D, Senner CE, Callery EM, Trotter MW, Hemberger M, Smith JC, et al. (2011). BRACHYURY and CDX2 mediate BMP-induced differentiation of human and mouse pluripotent stem cells into embryonic and extraembryonic lineages. Cell Stem Cell 9, 144-155.

Boland A, Chen Y, Raisch T, Jonas S, Kuzuoglu-Ozturk D, Wohlbold L, Weichenrieder O, Izaurralde E (2013). Structure and assembly of the NOT module of the human CCR4-NOT complex. Nat Struct Mol Biol 20, 1289-1297.

Bonet C, Giuliano S, Ohanna M, Bille K, Allegra M, Lacour JP, Bahadoran $P$, Rocchi S, Ballotti R, Bertolotto C (2012). Aurora B is regulated by the mitogen-activated protein kinase/extracellular signal-regulated kinase (MAPK/ERK) signaling pathway and is a valuable potential target in melanoma cells. J Biol Chem 287, 29887-29898. 
Burtscher I, Lickert H (2009). Foxa2 regulates polarity and epithelialization in the endoderm germ layer of the mouse embryo. Development 136, 1029-1038.

Carmena M, Wheelock M, Funabiki H, Earnshaw WC (2012). The chromosomal passenger complex (CPC): from easy rider to the godfather of mitosis. Nat Rev Mol Cell Biol 13, 789-803.

Caunt CJ, McArdle CA (2012). ERK phosphorylation and nuclear accumulation: insights from single-cell imaging. Biochem Soc Trans 40, 224-229

Cejas P, Cavazza A, Yandava CN, Moreno V, Horst D, Moreno-Rubio J, Burgos E, Mendiola M, Taing L, Goel A, et al. (2017). Transcriptional regulator $\mathrm{CNOT} 3$ defines an aggressive colorectal cancer subtype. Cancer Res 77, 766-779.

Chuai M, Hughes D, Weijer CJ (2012). Collective epithelial and mesenchymal cell migration during gastrulation. Curr Genomics 13, 267-277.

Chuderland D, Konson A, Seger R (2008). Identification and characterization of a general nuclear translocation signal in signaling proteins. Mol Cell 31, 850-861.

Ciruna B, Rossant J (2001). FGF signaling regulates mesoderm cell fate specification and morphogenetic movement at the primitive streak. Dev Cell 1, 37-49.

Collart MA, Panasenko OO, Nikolaev SI (2013). The Not3/5 subunit of the Ccr4-Not complex: a central regulator of gene expression that integrates signals between the cytoplasm and the nucleus in eukaryotic cells. Cell Signal 25, 743-751.

Costello I, Nowotschin S, Sun X, Mould AW, Hadjantonakis AK, Bikoff EK, Robertson EJ (2015). Lhx1 functions together with Otx2, Foxa2, and Ldb1 to govern anterior mesendoderm, node, and midline development. Genes Dev 29, 2108-2122.

De Keersmaecker K, Atak ZK, Li N, Vicente C, Patchett S, Girardi T, Gianfelici V, Geerdens E, Clappier E, Porcu M, et al. (2013). Exome sequencing identifies mutation in CNOT3 and ribosomal genes RPL5 and RPL10 in T-cell acute lymphoblastic leukemia. Nat Genet 45, 186-190.

Delacruz RGC, Sandoval IT, Chang K, Miller BN, Reyes-Uribe L, Borras E, Lynch PM, Taggart MW, Hawk ET, Vilar E, Jones DA (2019). Functional characterization of CNOT3 variants identified in familial adenomatous polyposis adenomas. Oncotarget 10, 3939-3951.

Deng CX, Wynshaw-Boris A, Shen MM, Daugherty C, Ornitz DM, Leder P (1994). Murine FGFR-1 is required for early postimplantation growth and axial organization. Genes Dev 8, 3045-3057.

Deng X, Ruvolo P, Carr B, May WS Jr (2000). Survival function of ERK1/2 as IL-3-activated, staurosporine-resistant Bcl2 kinases. Proc Natl Acad Sci USA 97, 1578-1583.

Dixon SE, Bhatti MM, Uversky VN, Dunker AK, Sullivan WJ Jr (2011). Regions of intrinsic disorder help identify a novel nuclear localization signal in Toxoplasma gondii histone acetyltransferase TgGCN5-B. Mol Biochem Parasitol 175, 192-195.

Domina AM, Vrana JA, Gregory MA, Hann SR, Craig RW (2004). MCL1 is phosphorylated in the PEST region and stabilized upon ERK activation in viable cells, and at additional sites with cytotoxic okadaic acid or taxol. Oncogene 23, 5301-5315.

Donchet A, Oliva J, Labaronne A, Tengo L, Miloudi M, Gerard FCA, Mas C, Schoehn G, Ruigrok RWH, Ducatez M, Crepin T (2019). The structure of the nucleoprotein of influenza D shows that all orthomyxoviridae nucleoproteins have a similar NPCORE, with or without a NPTAIL for nuclear transport. Sci Rep 9, 600

Forbes SA, Beare D, Boutselakis H, Bamford S, Bindal N, Tate J, Cole CG, Ward S, Dawson E, Ponting L, et al. (2017). COSMIC: somatic cancer genetics at high-resolution. Nucleic Acids Res 45, D777-D783.

Formstecher E, Ramos JW, Fauquet M, Calderwood DA, Hsieh JC, Canton B, Nguyen XT, Barnier JV, Camonis J, Ginsberg MH, Chneiweiss H (2001). PEA-15 mediates cytoplasmic sequestration of ERK MAP kinase. Dev Cell 1, 239-250.

Frangini A (2013). Characterization of the Role of Aurora B Kinase in Quiescent Lymphocytes. PhD Thesis. Imperial College London.

Fujiwara T, Dehart DB, Sulik KK, Hogan BL (2002). Distinct requirements for extra-embryonic and embryonic bone morphogenetic protein 4 in the formation of the node and primitive streak and coordination of left-right asymmetry in the mouse. Development 129, 4685-4696.

Georgopoulos NT, Kirkwood LA, Southgate J (2014). A novel bidirectional positive-feedback loop between Wnt-beta-catenin and EGFR-ERK plays a role in context-specific modulation of epithelial tissue regeneration. $J$ Cell Sci 127, 2967-2982.

Giannakis M, Mu XJ, Shukla SA, Qian ZR, Cohen O, Nishihara R, Bahl S, Cao Y, Amin-Mansour A, Yamauchi M, et al. (2016). Genomic correlates of immune-cell infiltrates in colorectal carcinoma. Cell Rep 15, 857-865.
Hornbeck PV, Zhang B, Murray B, Kornhauser JM, Latham V, Skrzypek E (2015). PhosphoSitePlus, 2014: mutations, PTMs and recalibrations. Nucleic Acids Res 43, D512-D520.

Hu G, Kim J, Xu Q, Leng Y, Orkin SH, Elledge SJ (2009). A genome-wide RNAi screen identifies a new transcriptional module required for selfrenewal. Genes Dev 23, 837-848.

James BP, Bunch TA, Krishnamoorthy S, Perkins LA, Brower DL (2007). Nuclear localization of the ERK MAP kinase mediated by Drosophila alphaPS2betaPS integrin and importin-7. Mol Biol Cell 18, 4190-4199.

Jeong W-J, Ro EJ, Choi K-Y (2018). Interaction between Wnt/ $\beta$-catenin and RAS-ERK pathways and an anti-cancer strategy via degradations of $\beta$-catenin and RAS by targeting the Wnt/ $\beta$-catenin pathway. NPJ Precis Oncol 2, 5.

Kim D, Pertea G, Trapnell C, Pimentel H, Kelley R, Salzberg SL (2013). TopHat2: accurate alignment of transcriptomes in the presence of insertions, deletions and gene fusions. Genome Biol 14, R36.

Kim JS, Kim BS, Kim J, Park CS, Chung IY (2010a). The phosphoinositide3-kinase/Akt pathway mediates the transient increase in Nanog expression during differentiation of F9 cells. Arch Pharm Res 33, 1117-1125.

Kim PT, Hoffman BG, Plesner A, Helgason CD, Verchere CB, Chung SW, Warnock GL, Mui AL, Ong CJ (2010b). Differentiation of mouse embryonic stem cells into endoderm without embryoid body formation. PLoS One 5, e14146.

Kolch W (2005). Coordinating ERK/MAPK signalling through scaffolds and inhibitors. Nat Rev Mol Cell Biol 6, 827-837.

Krens SF, Spaink HP, Snaar-Jagalska BE (2006). Functions of the MAPK family in vertebrate-development. FEBS Lett 580, 4984-4990.

Levchenko A, Bruck J, Sternberg PW (2000). Scaffold proteins may biphasically affect the levels of mitogen-activated protein kinase signaling and reduce its threshold properties. Proc Natl Acad Sci USA 97, 5818-5823.

Li X, Morita M, Kikuguchi C, Takahashi A, Suzuki T, Yamamoto T (2017). Adipocyte-specific disruption of mouse Cnot3 causes lipodystrophy. FEBS Lett 591, 358-368.

Liao Y, Smyth GK, Shi W (2013). The Subread aligner: fast, accurate and scalable read mapping by seed-and-vote. Nucleic Acids Res 41, e108.

Liao Y, Smyth GK, Shi W (2014). featureCounts: an efficient general purpose program for assigning sequence reads to genomic features. Bioinformatics (Oxford, England) 30, 923-930.

Love MI, Huber W, Anders S (2014). Moderated estimation of fold change and dispersion for RNA-seq data with DESeq2. Genome Biol 15, 550.

Mebratu Y, Tesfaigzi Y (2009). How ERK1/2 activation controls cell proliferation and cell death: is subcellular localization the answer? Cell Cycle 8, 1168-1175.

Mootha VK, Lindgren CM, Eriksson KF, Subramanian A, Sihag S, Lehar J, Puigserver P, Carlsson E, Ridderstrale M, Laurila E, et al. (2003). PGC1 alpha-responsive genes involved in oxidative phosphorylation are coordinately downregulated in human diabetes. Nat Genet 34, 267-273.

Neely GG, Kuba K, Cammarato A, Isobe K, Amann S, Zhang L, Murata M Elmen L, Gupta V, Arora S, et al. (2010). A global in vivo Drosophila RNAi screen identifies NOT3 as a conserved regulator of heart function. Cell 141, 142-153.

Neuwelt AJ, Kimball AK, Johnson AM, Arnold BW, Bullock BL, Kaspar RE, Kleczko EK, Kwak JW, Wu MH, Heasley LE, et al. (2020). Cancer cell-intrinsic expression of $\mathrm{MHC}$ II in lung cancer cell lines is actively restricted by MEK/ERK signaling and epigenetic mechanisms. J Immunother Cancer 8, e000441.

Priestley P, Baber J, Lolkema MP, Steeghs N, de Bruijn E, Shale C, Duyvesteyn K, Haidari S, van Hoeck A, Onstenk W, et al. (2019). Pancancer whole-genome analyses of metastatic solid tumours. Nature 575, 210-216.

Risso D, Ngai J, Speed TP, Dudoit S (2014). Normalization of RNA-seq data using factor analysis of control genes or samples. Nat Biotechnol 32, 896-902.

Rodriguez-Gil A, Ritter O, Saul W, Wilhelm J, Yang CY, Grosschedl R, Imai Y, Kuba K, Kracht M, Schmitz ML (2017). The CCR4-NOT complex contributes to repression of major histocompatibility complex class II transcription. Sci Rep 7, 3547

Roux PP, Blenis J (2004). ERK and p38 MAPK-activated protein kinases: a family of protein kinases with diverse biological functions. Microbiol Mol Biol Rev 68, 320-344.

Roy M, Li Z, Sacks DB (2004). IQGAP1 binds ERK2 and modulates its activity. J Biol Chem 279, 17329-17337.

Roy M, Li Z, Sacks DB (2005). IQGAP1 is a scaffold for mitogen-activated protein kinase signaling. Mol Cell Biol 25, 7940-7952.

Sacks DB (2006). The role of scaffold proteins in MEK/ERK signalling. Biochem Soc Trans 34, 833-836. 
Sebolt-Leopold JS, Dudley DT, Herrera R, Van Becelaere K, Wiland A, Gowan RC, Tecle H, Barrett SD, Bridges A, Przybranowski S, et al. (1999). Blockade of the MAP kinase pathway suppresses growth of colon tumors in vivo. Nat Med 5, 810-816.

Shirai YT, Mizutani A, Nishijima S, Horie M, Kikuguchi C, Elisseeva O, Yamamoto T (2019). CNOT3 targets negative cell cycle regulators in non-small cell lung cancer development. Oncogene 38, 2580-2594.

Simeone A (1998). Otx1 and Otx2 in the development and evolution of the mammalian brain. EMBO J 17, 6790-6798.

Simeone A, Acampora D, Mallamaci A, Stornaiuolo A, D'Apice MR, Nigro V Boncinelli E (1993). A vertebrate gene related to orthodenticle contains a homeodomain of the bicoid class and demarcates anterior neuroectoderm in the gastrulating mouse embryo. EMBO J 12, 2735-2747.

Sladitschek HL, Neveu PA (2019). A gene regulatory network controls the balance between mesendoderm and ectoderm at pluripotency exit. Mol Syst Biol 15, e9043.

Smith BN, Burton LJ, Henderson V, Randle DD, Morton DJ, Smith BA, Taliaferro-Smith L, Nagappan P, Yates C, Zayzafoon M, et al. (2014). Snail promotes epithelial mesenchymal transition in breast cancer cells in part via activation of nuclear ERK2. PLoS One 9, e104987.

Song J, Salek-Ardakani S, So T, Croft M (2007). The kinases aurora B and mTOR regulate the G1-S cell cycle progression of T lymphocytes. Nat Immunol 8, 64-73.

Stern CD, Downs KM (2012). The hypoblast (visceral endoderm): an evodevo perspective. Development 139, 1059-1069.

Stewart DJ (2014). Wnt signaling pathway in non-small cell lung cancer. J Natl Cancer Inst 106, djt356.

Subramanian A, Tamayo P, Mootha VK, Mukherjee S, Ebert BL, Gillette MA, Paulovich A, Pomeroy SL, Golub TR, Lander ES, Mesirov JP (2005). Gene set enrichment analysis: a knowledge-based approach for interpreting genome-wide expression profiles. Proc Natl Acad Sci USA 102, $15545-15550$.

Subramanian M, Shaha C (2007). Up-regulation of Bcl-2 through ERK phosphorylation is associated with human macrophage survival in an estrogen microenvironment. J Immunol 179, 2330-2338.

Sudhir PR, Hsu CL, Wang MJ, Wang YT, Chen YJ, Sung TY, Hsu WL, Yang UC, Chen JY (2011). Phosphoproteomics identifies oncogenic Ras signaling targets and their involvement in lung adenocarcinomas. PLoS One 6, e20199.

Sui L, Bouwens L, Mfopou JK (2013). Signaling pathways during maintenance and definitive endoderm differentiation of embryonic stem cells. Int J Dev Biol 57, 1-12.

Takeshita M, Koga T, Takayama K, ljichi K, Yano T, Maehara Y, Nakanishi Y, Sueishi K (2013). Aurora-B overexpression is correlated with aneuploidy and poor prognosis in non-small cell lung cancer. Lung Cancer 80, 85-90.

Tang YS, Lo CY, Mok CK, Chan PK, Shaw PC (2019). The extended C-terminal region of influenza $\mathrm{C}$ virus nucleoprotein is important for nuclear import and ribonucleoprotein activity. J Virol 93, e02048-18.

Trakala M, Fernandez-Miranda G, Perez de Castro I, Heeschen C, Malumbres M (2013). Aurora B prevents delayed DNA replication and premature mitotic exit by repressing p21(Cip1). Cell Cycle 12, 1030-1041.

Tremblay KD, Hoodless PA, Bikoff EK, Robertson EJ (2000). Formation of the definitive endoderm in mouse is a Smad2-dependent process. Development 127, 3079-3090.

Tsakiridis A, Huang Y, Blin G, Skylaki S, Wymeersch F, Osorno R, Economou C, Karagianni E, Zhao S, Lowell S, Wilson V (2014). Distinct Wnt-driven primitive streak-like populations reflect in vivo lineage precursors. Development 141, 1209-1221.
Tuncel $H$, Shimamoto F, Kaneko Guangying $Q i$, Aoki E, Jikihara H, Nakai S, Takata T, Tatsuka M (2012). Nuclear Aurora B and cytoplasmic survivin expression is involved in lymph node metastasis of colorectal cancer. Oncol Lett 3, 1109-1114.

Vallier L, Touboul T, Chng Z, Brimpari M, Hannan N, Millan E, Smithers LE, Trotter M, Rugg-Gunn P, Weber A, Pedersen RA (2009). Early cell fate decisions of human embryonic stem cells and mouse epiblast stem cells are controlled by the same signalling pathways. PLoS One 4, e6082.

Waese EY, Stanford WL (2011). One-step generation of murine embryonic stem cell-derived mesoderm progenitors and chondrocytes in a serumfree monolayer differentiation system. Stem Cell Res 6, 34-49.

Wang L, Chen YG (2016). Signaling control of differentiation of embryonic stem cells toward mesendoderm. J Mol Biol 428, 1409-1422.

Wang Z, Zhang B, Wang M, Carr BI (2003). Persistent ERK phosphorylation negatively regulates $C A M P$ response element-binding protein (CREB) activity via recruitment of CREB-binding protein to pp90RSK. J Biol Chem $278,11138-11144$

Warabi M, Kitagawa M, Hirokawa K (2000). Loss of MHC class II expression is associated with a decrease of tumor-infiltrating $T$ cells and an increase of metastatic potential of colorectal cancer: immunohistological and histopathological analyses as compared with normal colonic mucosa and adenomas. Pathol Res Pract 196, 807-815.

Willems $E$, Leyns $L$ (2008). Patterning of mouse embryonic stem cell-derived pan-mesoderm by Activin $\mathrm{A} / \mathrm{Nodal}$ and $\mathrm{Bmp} 4$ signaling requires fibroblast growth factor activity. Differentiation 76, 745-759.

Witzel F, Maddison L, Bluthgen N (2012). How scaffolds shape MAPK signaling: what we know and opportunities for systems approaches. Front Physiol 3, 475.

Wolfe AD, Downs KM (2014). Mixl1 localizes to putative axial stem cell reservoirs and their posterior descendants in the mouse embryo. Gene Expr Patterns 15, 8-20.

Xing J, Kornhauser JM, Xia Z, Thiele EA, Greenberg ME (1998). Nerve growth factor activates extracellular signal-regulated kinase and p38 mitogen-activated protein kinase pathways to stimulate CREB serine 133 phosphorylation. Mol Cell Biol 18, 1946-1955.

Yamaguchi TP, Harpal K, Henkemeyer M, Rossant J (1994). fgfr-1 is required for embryonic growth and mesodermal patterning during mouse gastrulation. Genes Dev 8, 3032-3044.

Yang CY, Ramamoorthy S, Boller S, Rosenbaum M, Rodriguez Gil A, Mittler G, Imai Y, Kuba K, Grosschedl R (2016). Interaction of CCR4-NOT with EBF1 regulates gene-specific transcription and mRNA stability in B lymphopoiesis. Genes Dev 30, 2310-2324.

Yao Y, Li W, Wu J, Germann UA, Su MS, Kuida K, Boucher DM (2003). Extracellular signal-regulated kinase 2 is necessary for mesoderm differentiation. Proc Natl Acad Sci USA 100, 12759-12764.

Young MD, Wakefield MJ, Smyth GK, Oshlack A (2010). Gene ontology analysis for RNA-seq: accounting for selection bias. Genome Biol 11, R14.

Yu P, Pan G, Yu J, Thomson JA (2011). FGF2 sustains NANOG and switches the outcome of BMP4-induced human embryonic stem cell differentiation. Cell Stem Cell 8, 326-334.

Zhan T, Rindtorff N, Boutros M (2017). Wnt signaling in cancer. Oncogene $36,1461-1473$

Zhang Y, Jiang C, Li H, Lv F, Li X, Qian X, Fu L, Xu B, Guo X (2015). Elevated Aurora $B$ expression contributes to chemoresistance and poor prognosis in breast cancer. Int J Clin Exp Pathol 8, 751-757.

Zheng X, Dumitru R, Lackford BL, Freudenberg JM, Singh AP, Archer TK, Jothi R, Hu G (2012). Cnot1, Cnot2, and Cnot3 maintain mouse and human ESC identity and inhibit extraembryonic differentiation. Stem Cells (Dayton, Ohio) , 910-922. 


\section{Supplemental Materials}

Molecular Biology of the Cell

Sarkar et al. 
Sarkar et al.

\section{SUPPLEMENTAL INFORMATION}




\section{Figure S1}

A

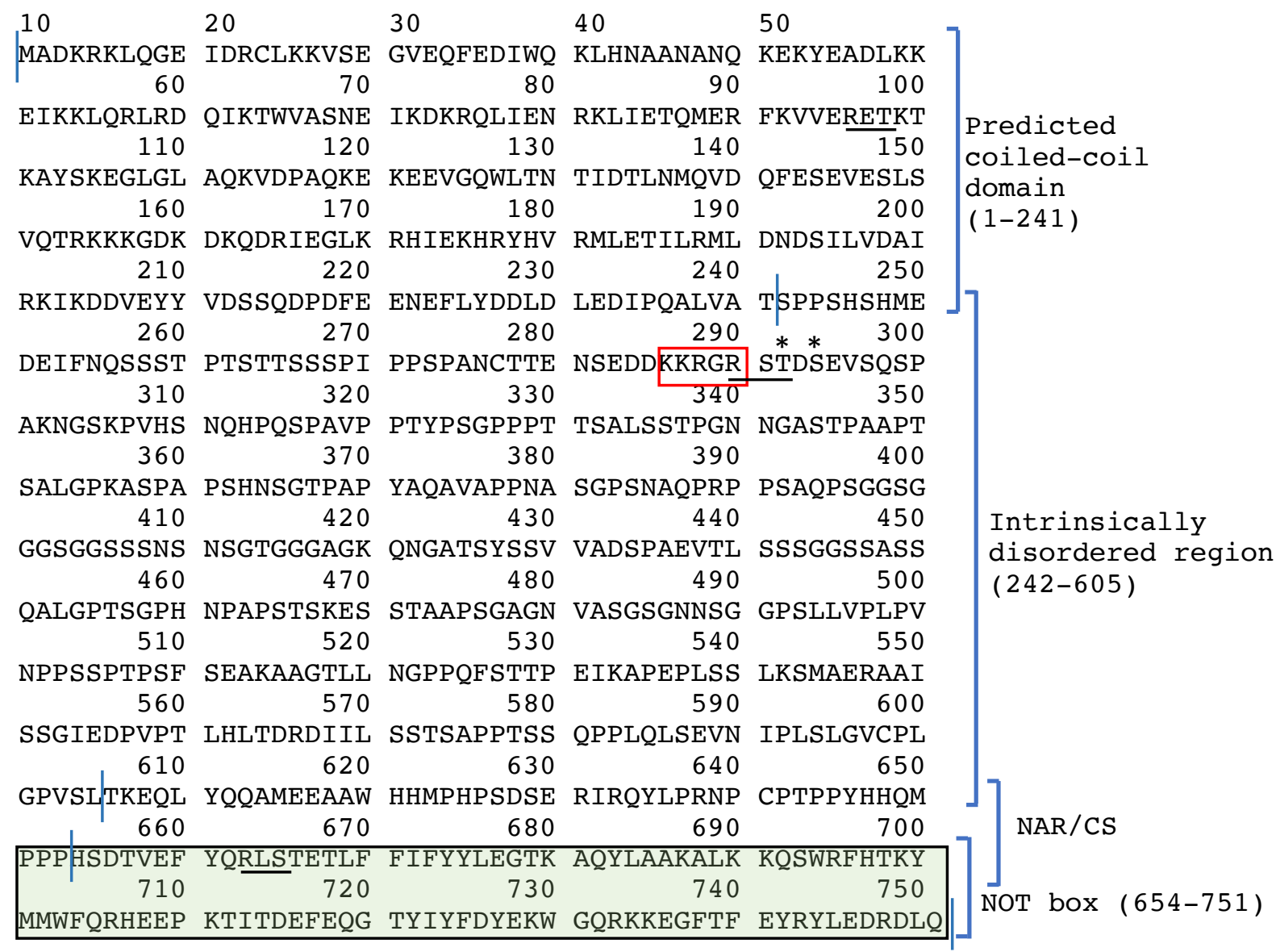

B

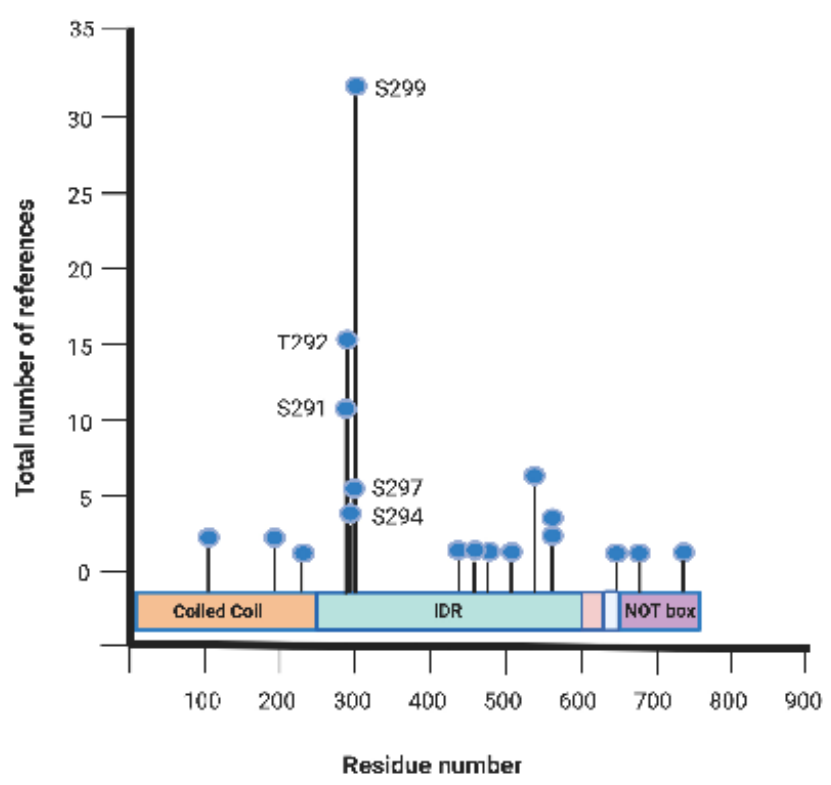


Supplementary Figure 1: A. Sequence of the mouse CNOT3 protein (UNIPROT Q8K0V4). Vertical blue lines delineate the domains of the protein that are shown in Figure 1B. The green-shaded box indicates the the NOT box region, which is required for the interaction between CNOT3 and Aurora B (Figure 1C). Aurora B consensus phosphorylation sites are underlined. The red box and red text indicate the putative nuclear localization sequence. * indicates residues 292 and 294, which were mutated in this study.

B. Phosphorylation sites detected on the CNOT3 protein by in vivo proteomic discovery mass pectrometry. Data was obtained from PhosphoSite ${ }^{\circledR}$ (Hornbeck et al., 2015). The data shown is for the human CNOT3 protein, which is $95 \%$ identical to mouse CNOT3. With the exception of one residue (E79), all of the residues that vary between human and mouse are located within the intrinsically disordered region between residues 329 and 604 of the mouse sequence. Blue circles indicate phosphorylated residues. The yaxis shows the number of records where the modification was assigned using proteomic discovery mass spectrometry. The numbered residues show a phosphorylation hotspot that extends from positions 291-299. 


\section{Figure S2}

A
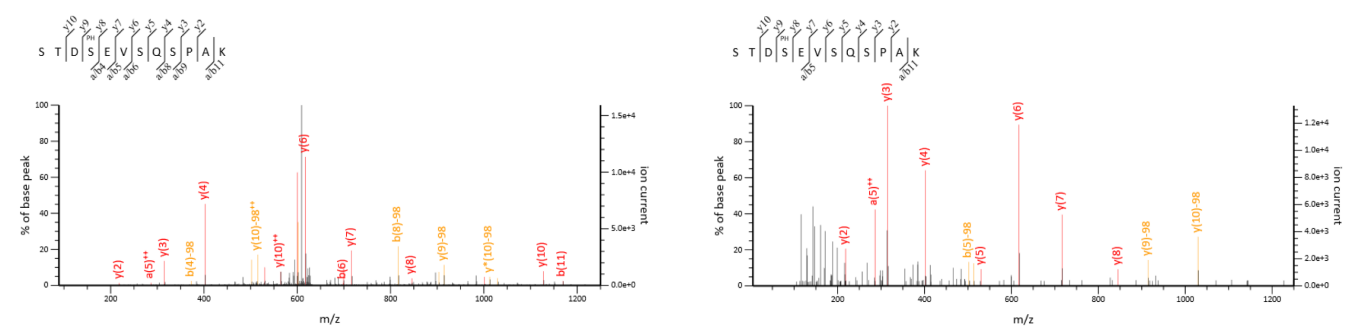

B

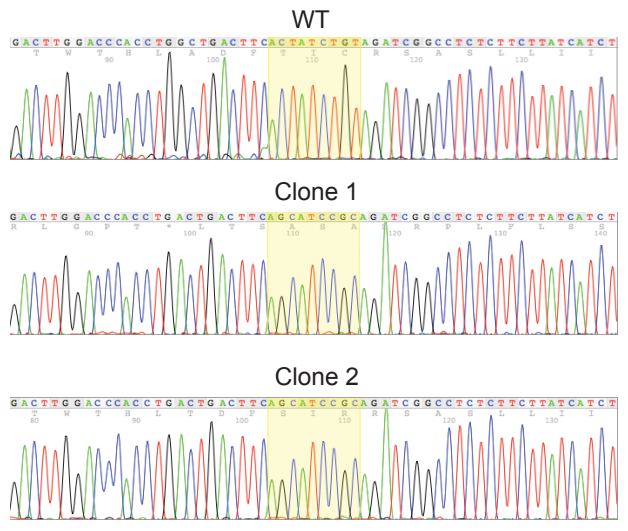

C
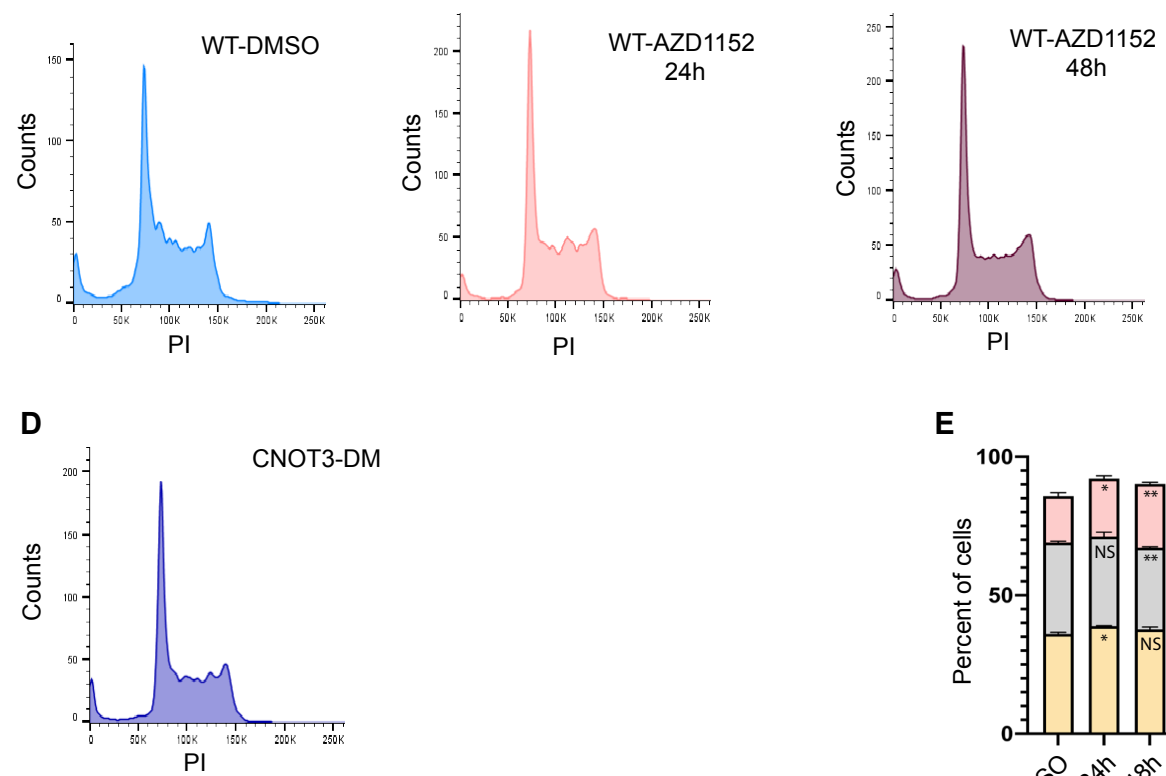

E
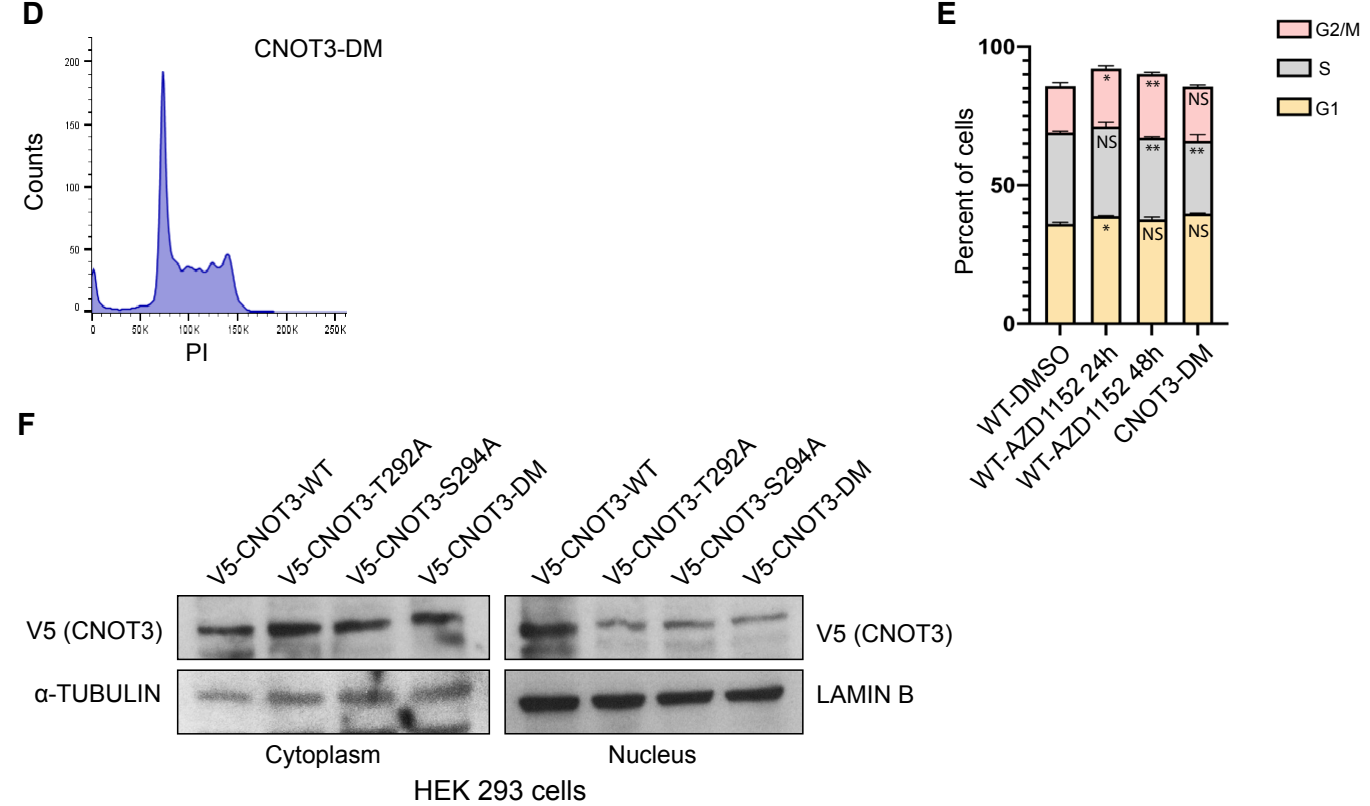

HEK 293 cells 


\section{Supplementary Figure 2: Identification of Aurora B phosphorylation sites in the CNOT3 protein}

A. MS/MS fragmentation spectrum generated by ion trap collision-induced dissociation (CID) of doubly charged precursor ion with $\mathrm{m} / \mathrm{z} 658.27$ identifying CNOT3 tryptic peptide 291-302 (STDSEVSQSPAK). The spectrum presented two fragments which were as follows: Left panel: query number 2609 with assigned b- and y-ions; MASCOT score: 54; expectation value: $1.8 \times 10^{-5}$; neutral loss of $\mathrm{H}_{3} \mathrm{PO}_{4}$ (-97.98 Da) from precursor ion detected at m/z 609.49; localisation probability for phosphorylation of Ser291: 3.0\%, Thr292: 3.0\%, Ser294 is 93.9\%. Right panel: query number 2608 with assigned b- and y-ions; MASCOT score: 53; expectation value: 3.6x10-5; localisation probability for phosphorylation of Ser291: 25.6\%, Thr292: 25.6\%, Ser294: 48.4\%.

B. Left panel: Schematic representation showing use of CRISPR/Cas9 targeting of Cnot3 in exon 10 to mutate Threonine 292 and Serine 294 to alanine, using the guide RNA described in Methods and a 110 bp single-stranded donor oligonucleotides (ssODN) carrying the mutations. Right panel: Sequence images showing the wild-type (WT) sequence and the mutated sequences obtained from two CRISPR generated clones. C. FACS analysis of the cell cycle profiles of ESCs incubated for 24 and 48 hours with the Aurora B inhibitor AZD1152 or with vehicle (DMSO) and stained with propidium iodide (PI).

D. FACS analysis of the cell cycle profile of Cnot3-DM ESCs.

E. Histograms showing the percentage of cells measured at different phases of the cell cycle in (c) and (d). Mean \pm SEM; P values (with respect to WT-DMSO) calculated by unpaired $t$-test, ns $=$ non-significant, $n=3$.

F. Representative immunoblot analysis of V5-tagged CNOT3 carried out on cytoplasmic and nuclear extracts from HEK 293 cells transfected with WT Cnot3, Cnot3-T292A, Cnot3-S294A, or Cnot3-DM expression constructs. Cells were harvested 48 hours posttransfection. $\alpha$-Tubulin and Lamin B were used as loading controls for the cytoplasm and nucleus respectively. 


\section{Figure S3}

A

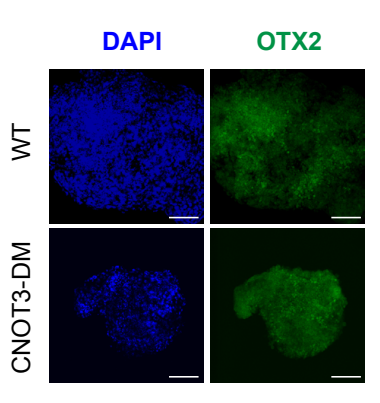

B

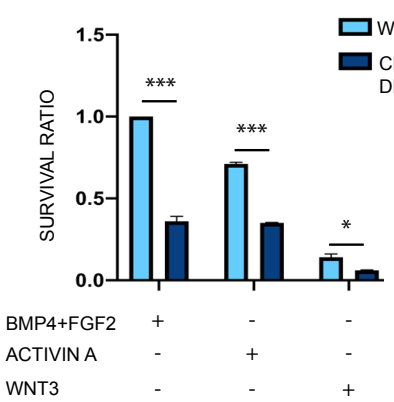

C

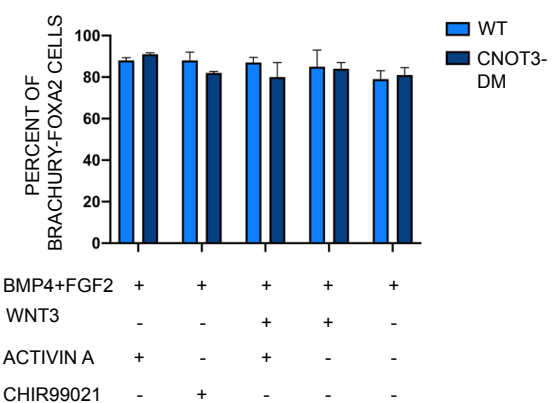

D
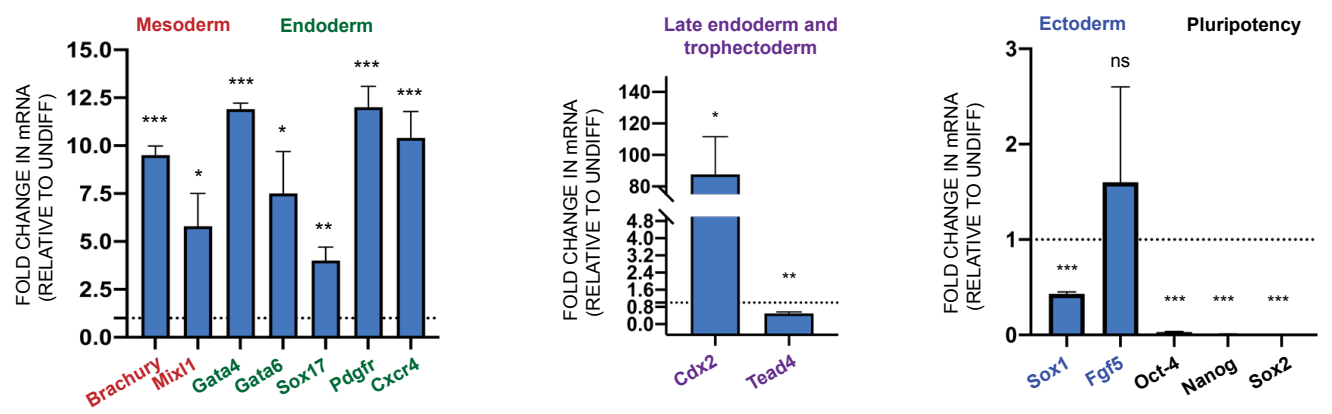

Lineage marker expression in differentiated wild-type (WT) mesendodermal cells 4 days mesendoderm differentiation

Supplementary Figure 3: Differentiation and survival of Cnot3-DM mesendoderm A. Representative confocal images of 8-day embryoid bodies (EBs) derived from wildtype (WT) and Cnot3-DM ESCs and subjected to immunostaining for 0TX2 (green). Nuclei were counterstained with DAPI; scale bar: $100 \mu \mathrm{m}$.

B. Survival of WT and Cnot3-DM ESCs over 4 days of differentiation induced in the presence of BMP4+FGF2 or Activin A or Wnt3 and the cell survival was determined using WST-1 reagent on day 4 of the differentiation. Histograms show survival ratios calculated relative to the values obtained for wild-type (WT) cells treated with BMP4+FGF2, which was assigned a value of 1 . Mean \pm SEM, significance calculated using unpaired t-test $* \mathrm{P}<0.05,{ }^{* * *} \mathrm{P}<0.001, \mathrm{n}=3$.

C. Histogram shows percentage of Brachyury and FOXA2 positive cells in the WT and Cnot3-DM ESCs treated with different combinations of the ligands used in (Figure 3C) over 4 days of ME differentiation. Dual positive cells for Brachyury and FOXA2 were quantified from randomly chosen fields from at least 100 cells for each biological replicate and plotted as percent of the total number of nuclei (DAPI) for WT and Cnot3DM ESCs in each case. Significance was calculated between WT and Cnot3-DM for each combination using unpaired $t$-test, ns:non-significant, $n=2$.

D. Analysis of mRNA levels of lineage marker genes after BMP4+FGF2 induced differentiation of WT ESCs for 4 days. Values for the undifferentiated (undiff) ESCs 
were set at 1 as indicated by a horizontal line in each histogram. $C d x 2$ is a marker for late endoderm and trophectoderm. Tead4 is a marker for early trophectoderm. Mean \pm SEM; $n=3$. $\mathrm{P}$ values are calculated by unpaired $t$-test, ${ }^{*} \mathrm{P}<0.05,{ }^{* *} \mathrm{P}<0.01,{ }^{* * *} \mathrm{P}<0.001$, ns $=$ non significant. 


\section{Figure S4}

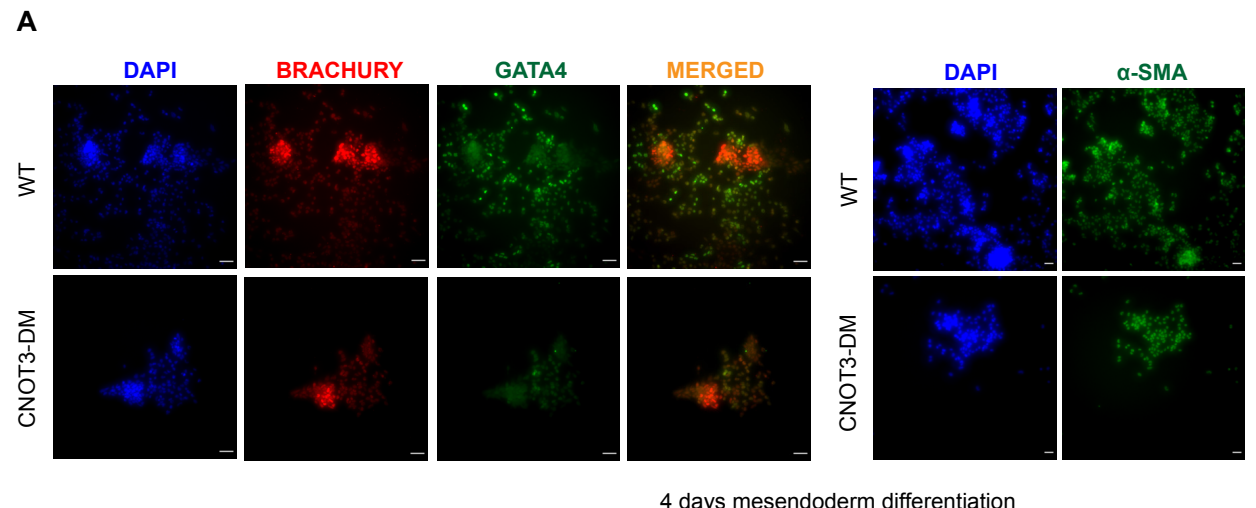

B
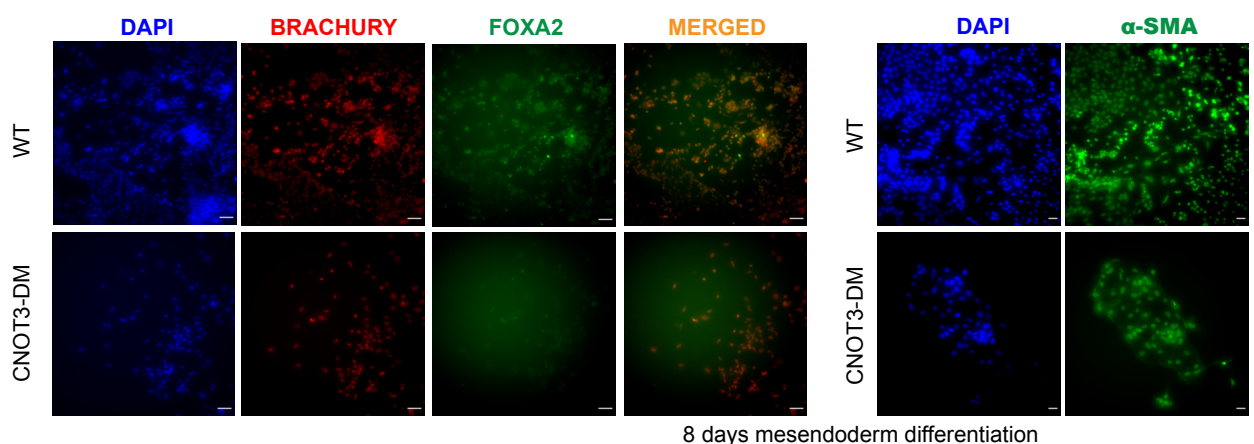

8 days mesendoderm differentiation

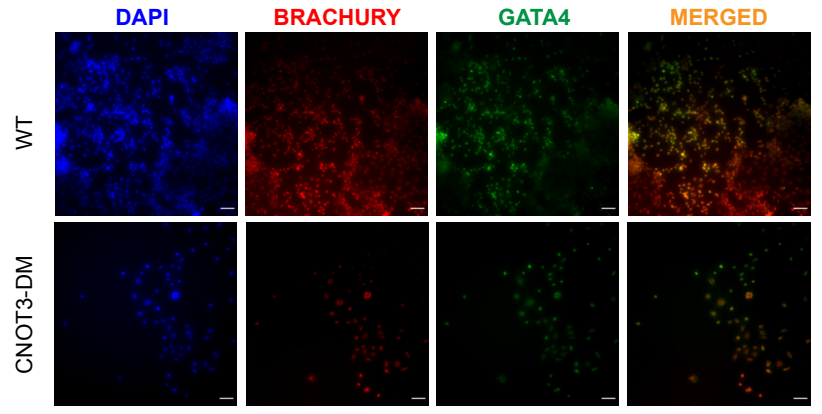

8 days mesendoderm differentiation

C

D
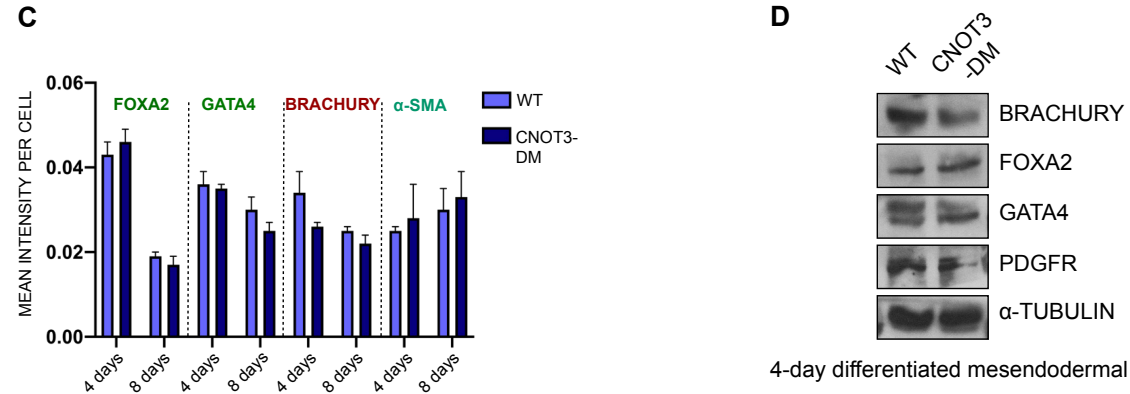

4-day differentiated mesendodermal cells 


\section{Supplementary Figure 4: CNOT3-T292/S294 phosphorylation promotes survival of differentiating mesendodermal cells.}

A. Staining for ME markers shows that differentiation of Cnot3-DM ESCs with BMP4 + FGF2 for 4 days generates reduced numbers of ME cells. Left panel: Representative IF analyses of staining of differentiated cells for the Brachury (red) and GATA4 (green) carried out on ME cells differentiated from WT and Cnot3-DM ESCs by incubating them with BMP4 + FGF2. Merged red and green images show the ME cells. Scale bars: $100 \mu \mathrm{m}$. Right panel: staining for smooth muscle actin (SMA) (green, scale bar: $60 \mu \mathrm{m}$ ). Nuclei were stained with DAPI.

B. Representative immunofluorescence images of ME cells generated by inducing differentiation of WT and Cnot3-DM ESCs with BMP4 + FGF2 for 8 days (left panel: scale bar: $100 \mu \mathrm{m}$ ). Top left panel: staining for Brachury (red) and FOXA2 (green). Bottom panel: staining for Brachyury (red) and GATA4 (green). Merged images show dual staining of the mesendodermal cells. Top right panel: staining for $\alpha$-SMA (scale bar: 60 $\mu \mathrm{m})$. Nuclei were counterstained with DAPI.

C. Histogram shows expression of the indicated markers in the WT and Cnot3-DM ESCs treated with BMP4 + FGF2 for 4 days and 8 days (A and B) of ME differentiation.

Expression for each marker is plotted as mean intensity per cell and was quantified from randomly chosen fields from at least 100 cells for each biological replicate for WT and Cnot3-DM ESCs in each case. Significance was calculated between WT and Cnot3DM using unpaired $t$-test, ns:non-significant, $n=3$.

D. Representative immunoblot analysis of the indicated proteins carried out on cell extracts from WT and Cnot3-DM ESCs subjected to 4 days of BMP4 + FGF2 induced differentiation into ME. Loading control: $\alpha$-Tubulin. 


\section{Figure S5}

A
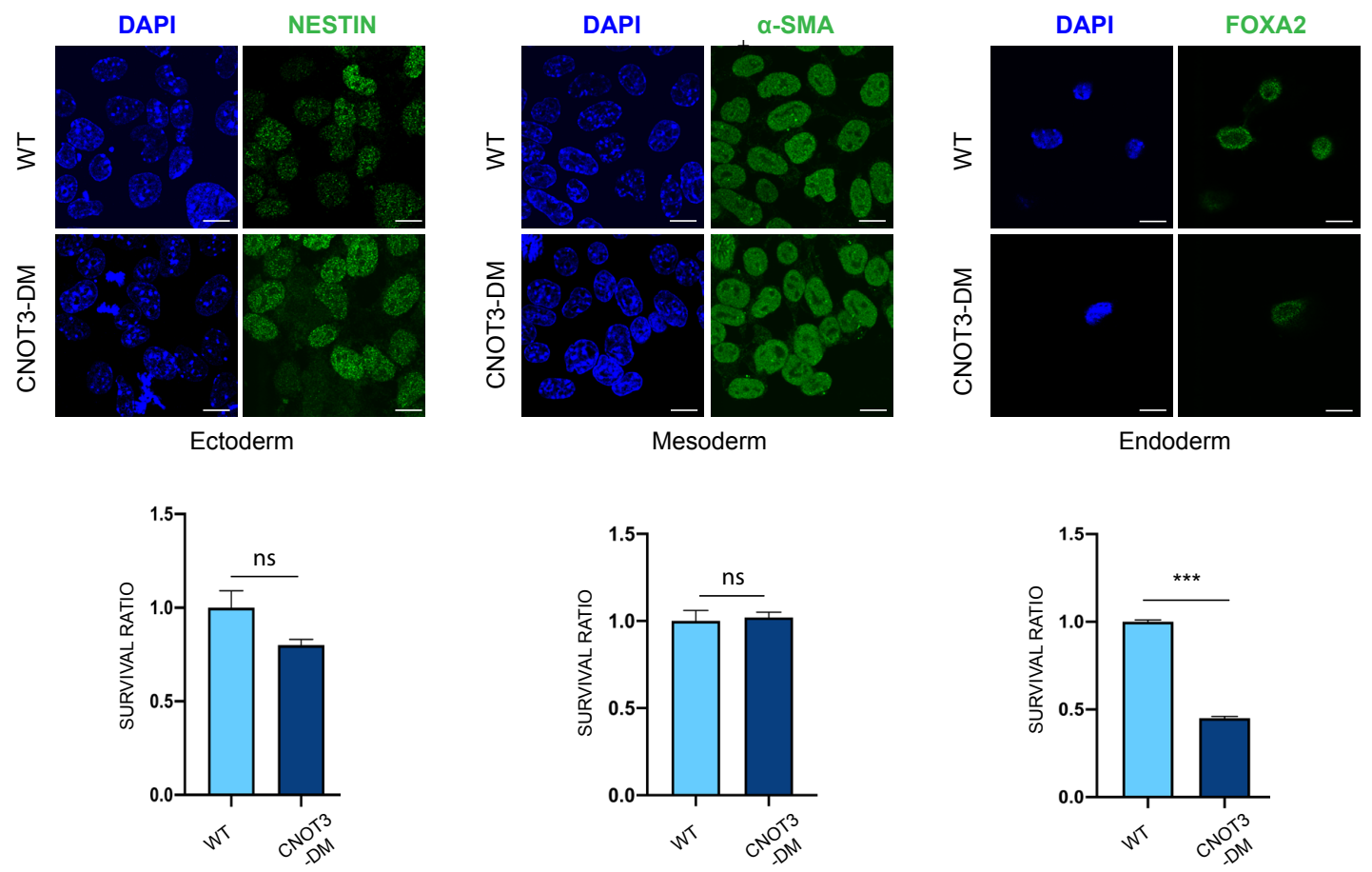

B

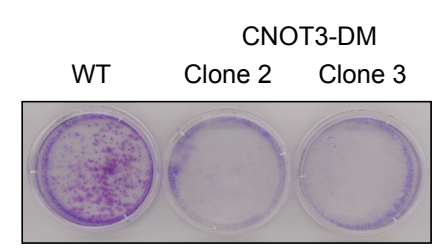

8 days mesendoderm differentiation
C

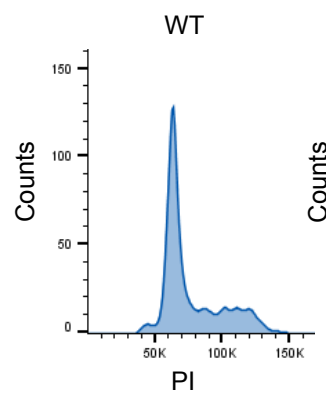

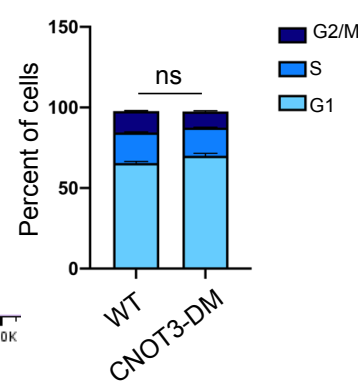

D

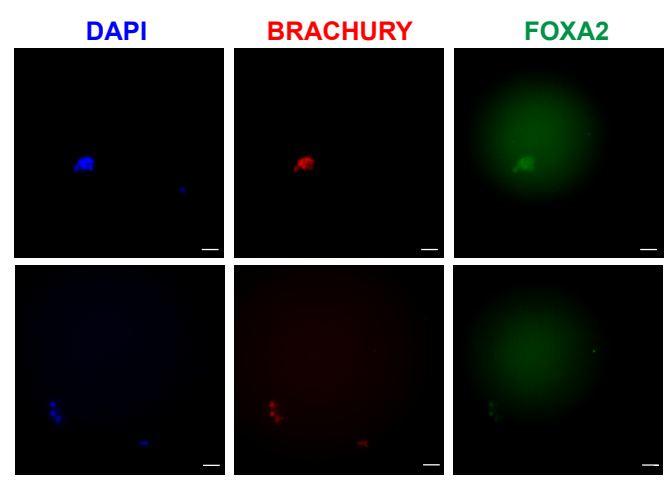

MERGED
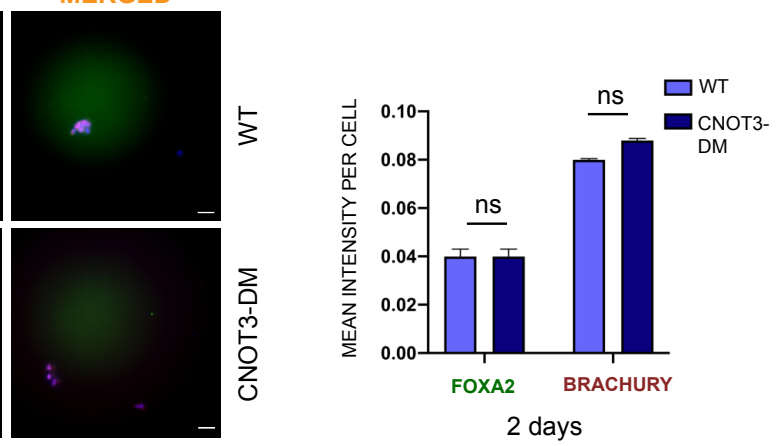

2 days mesendoderm differentiation 


\section{Supplementary Figure 5: Differentiation of Cnot3-DM ESCs into the three germ layers.}

A. Top panels: Representative confocal images of differentiation of wild-type (WT) and Cnot3-DM ESCs into ectoderm (3 days; immunostained for Nestin (green)), Activin A induced mesoderm ( 3 days; immunostained for $\alpha$-SMA (green)) and FGF2+retinoic acid induced endoderm (3 days; immunostained for FOXA2 (green). Nuclei were counterstained with DAPI; scale bar: $12 \mu \mathrm{m}$. (See Methods). Bottom panels: Graphs represent the ratio of suriving Cnot3-DM cells versus WT cells after 3 days of differentiation into each lineage. Cell survival was measured using WST-1 reagent (see Methods). Mean \pm SEM; (P values calculated by unpaired t-test, ${ }^{* * *} \mathrm{P}<0.001$. ns $=$ nonsignificant, $\mathrm{n}=3$ ).

B. Crystal violet staining showing the efficiency of BMP4+FGF2 induced mesendoderm (ME) differentiation of wild-type (WT) ESCs and two additional clones of Cnot3-DM ESCs after 4 days of differentiation.

C. Cell cycle analysis of ME cells obtained by inducing differentiation of WT and Cnot3DM ESCs with BMP4 + FGF2 for 4 days. Histograms represent the percentage of cells at different phases of the cell cycle. Mean \pm SEM; P values calculated by unpaired $t$-test, $n s$ $=$ non-significant, $n=3$.

D. Representative immunofluorescence images of ME cells generated by inducing differentiation of WT and Cnot3-DM ESCs with BMP4 + FGF2 for 2 days (left panel: scale bar: $100 \mu \mathrm{m}$ ). Staining was for Brachury (red) and FOXA2 (green). Merged images show dual staining of the mesendodermal cells. Nuclei were counterstained with DAPI. Right panel: Histogram shows levels of staining for FOXA2 and Brachyury in the WT and Cnot3-DM ESCs treated with BMP4 + FGF2 for 2 days (left panel) of ME differentiation. Expression for each marker is plotted as mean intensity per cell and was quantified from randomly chosen fields from at least 30 cells for each biological replicate for WT and Cnot3-DM ESCs in each case. Significance was calculated between WT and Cnot3DM using unpaired $t$-test, ns:non-significant, $n=2$. 


\section{Figure S6}

A

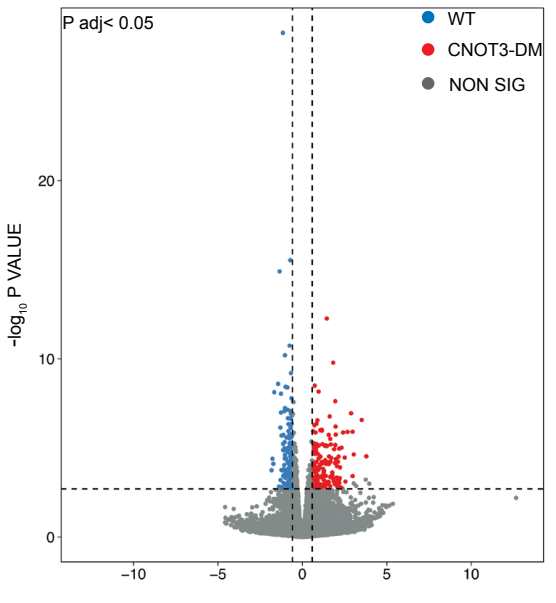

$-\log _{2}$ FOLD CHANGE

C

ENRICHED GENE ONTOLOGY TERMS (UPREGULATED)

Epithelium development

Mesenchymal cell development

Embryonic placenta development

Negative regulation of Wnt signaling pathway

Apoptotic process involved in morphogenesis

Regulation of programmed cell death $\begin{array}{llllll}2 & 4 & 6 & 8 & 10 & 12\end{array}$

E

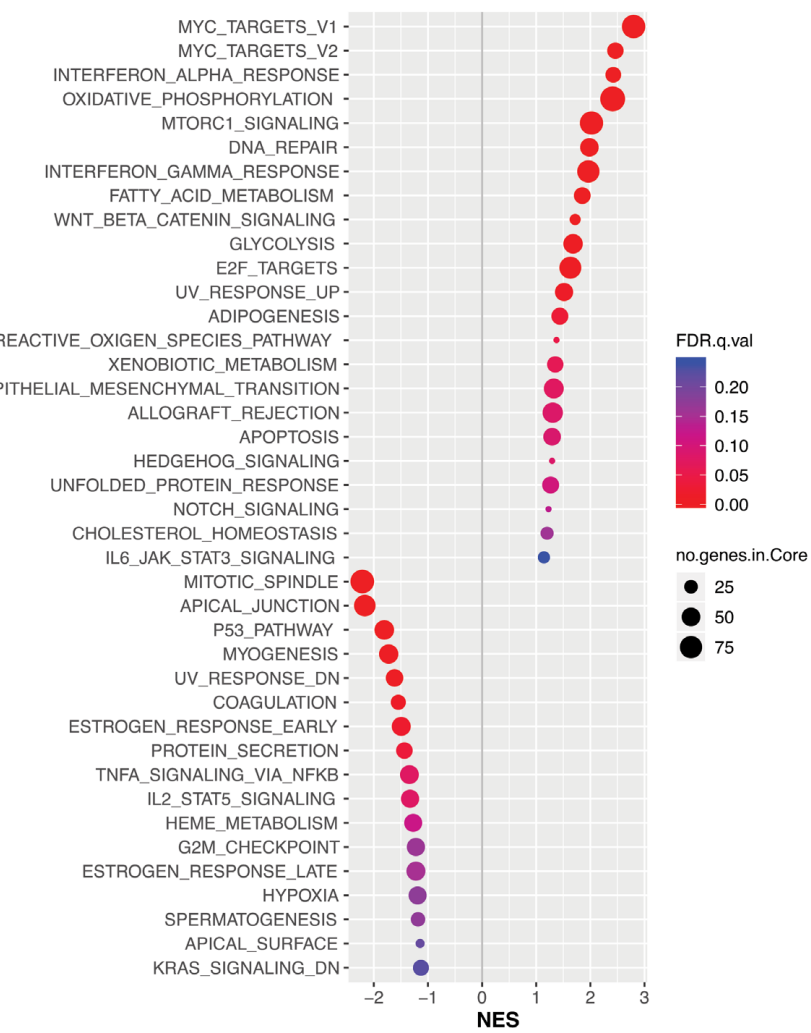

B

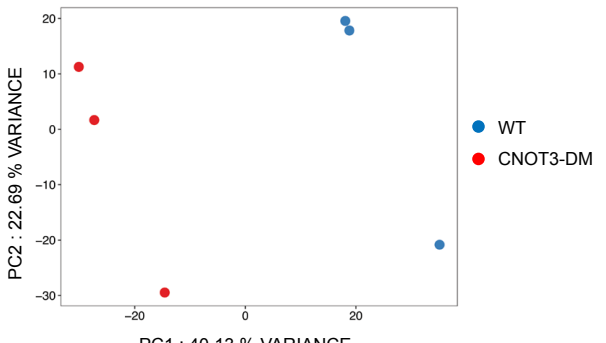

PC1 : $40.13 \%$ VARIANCE

D

ENRICHED GENE ONTOLOGY TERMS (DOWNREGULATED)

Cell adhesion

Developmental processes

Signal transducer activity

Molecular transducer activity

Signaling receptor activity

Cell surface receptor signaling pathway

Growth factor binding

Regulation of cell proliferation

Positive regulation of kinase activity

Multicellular organism developmen

Regulation of MAPK cascade

$-\log _{10}$ P VALUE 


\section{Supplementary Figure 6: Transcriptome profiling by RNA sequencing in differentiating wild-type and Cnot3-DM mesendoderm cells.}

A. and B. RNA-seq analysis was used to compare gene expression patterns of ME cells obtained by differentiating wild-type (WT) and Cnot3-DM ESCs for 4 days with BMP4 + FGF2.

A. Volcano plot of RNA-Seq transcriptome data showing differentially expressed genes after 4 days of BMP4 + FGF2 induced mesendoderm differentiation of WT and Cnot3DM ESCs. Fold change ratio $>1.5$; adjusted $\mathrm{P}$ value $<0.05$. Three biological replicates for each were analyzed.

B. Principal component plot of the RNA-seq data showing PC1 and PC2 and the percent variance obtained for each. This reveals separate clustering of the genes that are differentially expressed between the WT and Cnot3-DM-derived 4-day differentiated mesendodermal cells.

C. and D. Selected gene ontology (GO) profiles obtained out using the GOSeq Bioconductor package and showing upregulated genes (C) and downregulated genes (D), which are involved in signalling pathways that have key roles in developmental processes. Adjusted $\mathrm{P}$ value $<0.05$.

E. GSEA analysis showing Hallmark gene sets that were significantly altered in the differentiated cells. See also Supplementary Tables S3A and S3B for the differentially regulated genes. The GO and GSEA analysis both showed that expression of genes involved in cell death and apoptosis was upregulated ( $\mathrm{C}$ and $\mathrm{E}$ ). The negative regulator of Wnt signalling Axin2, and TGF $\beta$, which is part of the apoptosis program, were also elevated. Additionally, the GO analysis revealed downregulation of gene expression programs that are involved in cell adhesion and developmental processes (D). The changes included downregulation of signalling molecules such as Wnt6. Bcl2, a key cell survival factor downstream of ERK signalling was also downregulated. 


\section{Figure S7}

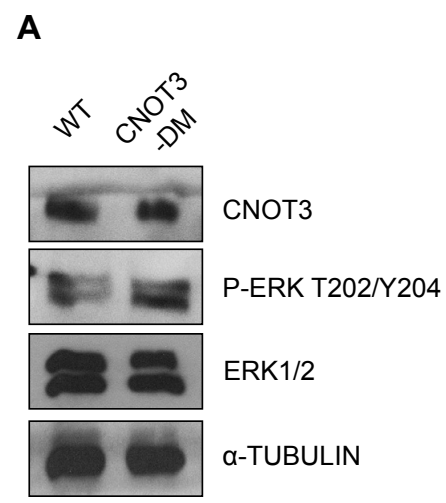

Undifferentiated ES cells

B

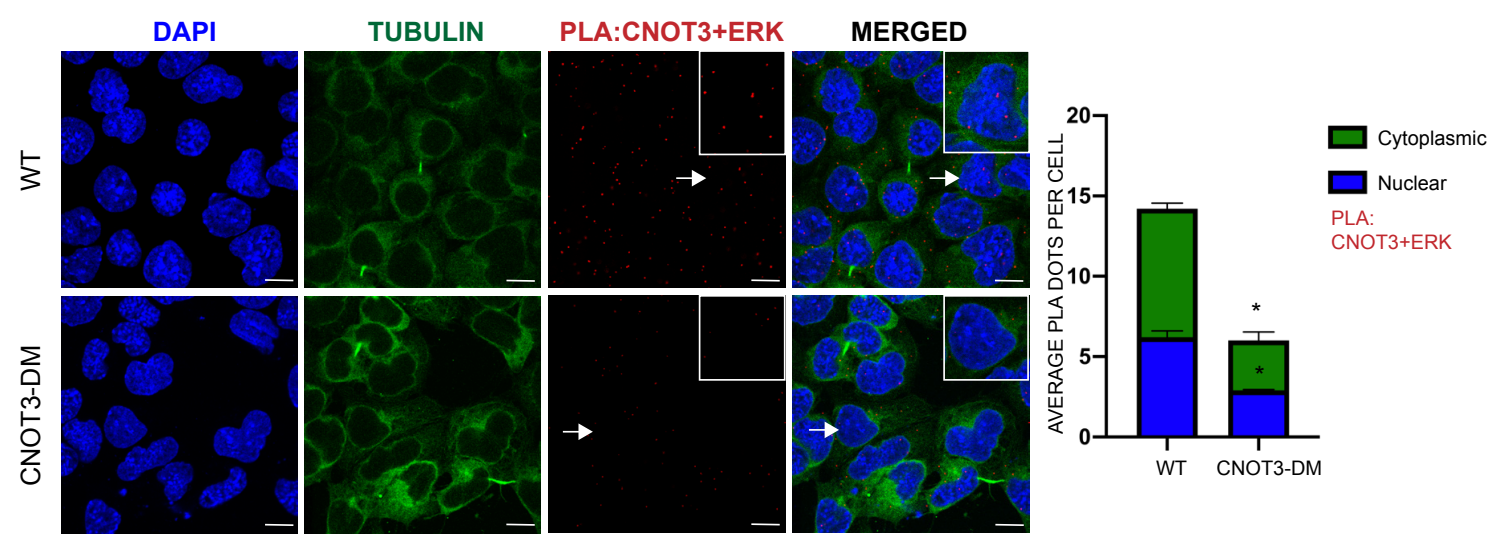

C

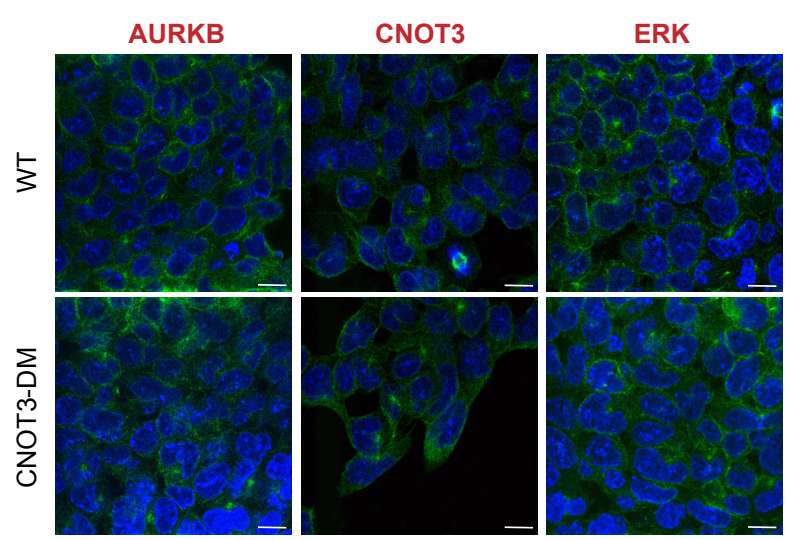

Supplementary Figure 7: Phosphorylation of CNOT3-T292/S294 alters interaction of CNOT3 with ERK in mesendodermal cells.

A.Immunoblotting analysis of the indicated proteins from cell extracts prepared from undifferentiated wild-type (WT) and Cnot3-DM ESCs. $\alpha$-Tubulin was used as a loading control. 
B. PLA detection of endogenous interaction between CNOT3 and ERK using a second pair of antibodies (results from the first antibody pair are shown in Figure 7B). WT and Cnot3-DM ESCs were induced to differentiate into mesendodermal cells by incubation for 4 days with BMP4 + FGF2. Positive PLA signals are visible as red dots. Nuclei were counterstained with DAPI and staining for Tubulin was used to mark the cell cytoplasm. Boxed areas represent an enlarged cell indicated by the arrows; scale bar: $10 \mu \mathrm{m}$. PLA dots were quantified from randomly chosen fields from at least 50 cells for each biological replicate. Histogram represents average interactions per cell (dots per cell) as well as the nuclear and cytoplasmic distribution. Error bars represent mean \pm SEM; $n=2$. (P values calculated by unpaired $t$-test, ${ }^{*} \mathrm{P}<0.05$ ).

C. Negative control single PLAs for analysis shown in Figure 6. The control assays were conducted with conducted with anti-Aurora B only, anti-CNOT3 only or anti-ERK only. 


\section{Supplementary Table 1}

List of primers used for qPCR

\begin{tabular}{|l|l|}
\hline Oct-4 & $\begin{array}{l}\text { F-5'-CCAATCAGCTTGGGCTAGAG-3' } \\
\text { R-5'-CTGGGAAAGGTGTCCCTGTA-3' }\end{array}$ \\
\hline Nanog & F-5'-TACCTCAGCCTCCAGCAGAT-3' \\
& R-5'-GCAATGGATGCTGGGATACT-3' \\
\hline Sox2 & F-5'-CACAACTCGGAGATCAGCAA-3' \\
& R-5'-CTCCGGGAAGCGTGTACTTA-3' \\
\hline Sox1 & F-5'-CCTCGGATCTCTGGTCAAGT-3' \\
& R-5'-TACAGAGCCGGCAGTCATAC-3' \\
\hline Fgf5 & F-5'-TCTGGATCTCCTTTGCGTTT-3' \\
& R-5'-GGGCTTCGAAAGCACATTTA-3' \\
\hline Cdx2 & F-5'-TGGTGTACACAGACCATCAGC-3' \\
& R-5'-CCTTGGCTCTGCGGTTCT-3' \\
\hline Tead4 & F-5'-ATCCTGACGGAGGAAGGCA-3' \\
& R-5'-GCTTGATATGGCGTGCGAT-3' \\
\hline Brachury & F-5'-CAGCTGTCGGGGAGCCTGG-3' \\
& R-5'-TGCTGCCTGTGAGTCATAAC-3' \\
\hline Mixl1 & F-5'-AGTTGCTGGAGCTCGTCTTC-3' \\
& R-5'-TTCTGGAACCACACCTGGAT-3' \\
\hline Gata4 & F-5'-TTCCTCTCCCAGGAACATCAA-3' \\
& R-5'-GCTGCACAACTGGGCTCTACTT-3' \\
\hline Gata6 & F-5'-ACAGCCCACTTCTGTGTTCCC-3' \\
& R-5'-GTGGGTTGGTCACGTGGTACAG-3' \\
\hline Sox17 & F-5'- TTCTGTACACTTTAATGAGGCTGTTC-3' \\
& R-5'-TTGTGGGAAGTGGGATCAAG-3' \\
\hline Pdgfr & F-5'-CTGGTGCCTGCCTCCTATGAC-3' \\
& R-5'-CACGATCGTTTCTCCTGCCTTAT-3' \\
\hline Cxcr4 & F-5'-GAGGCCAAGGAAACTGCTG-3' \\
& R-5'-GCGGTCACAGATGTACCTGTC-3' \\
\hline F-5'-ATGACGATGAGGATCGGAAAAAC-3' & R-5'-GTCCCCTTGGGTCGGACTTC-3' \\
& \\
\hline
\end{tabular}




\section{Supplementary Table 2}

List of antibodies

\begin{tabular}{|c|c|c|}
\hline Antibody & Source & Catalouge number \\
\hline Rabbit polyclonal anti-H3 & ABCAM & Cat\# ab1791 \\
\hline Rabbit polyclonal anti-CNOT3 & Bethyl Laboratories,Inc & Cat\# A302-156A \\
\hline Mouse monoclonal anti-CNOT3 & Abnova & $\begin{array}{l}\text { Cat\# H00004849- } \\
\text { M01 }\end{array}$ \\
\hline Mouse monoclonal anti-ERK1/2 & $\begin{array}{l}\text { Santa Cruz } \\
\text { Biotechnology }\end{array}$ & Cat\# sc-514302 \\
\hline Rabbit polyclonal anti-ERK1/2 & Cell Signaling Technology & Cat\# 9102 \\
\hline $\begin{array}{l}\text { Rabbit polyclonal anti-phospho- } \\
\text { ERK1/2(T202/Y204) }\end{array}$ & Cell Signaling Technology & Cat\# 9101 \\
\hline Mouse monoclonal anti-Aurora B & $\begin{array}{l}\text { Santa Cruz } \\
\text { Biotechnology }\end{array}$ & Cat\# sc-393357 \\
\hline Mouse monoclonal anti-Lamin B1 & $\begin{array}{l}\text { Santa Cruz } \\
\text { Biotechnology }\end{array}$ & Cat\# sc-374015 \\
\hline Mouse monoclonal anti- $\alpha$ Tubulin & $\begin{array}{l}\text { Santa Cruz } \\
\text { Biotechnology }\end{array}$ & Cat\# sc-5286 \\
\hline Mouse monoclonal anti-0TX2 & $\begin{array}{l}\text { Santa Cruz } \\
\text { Biotechnology }\end{array}$ & Cat\# sc-514195 \\
\hline Rabbit polyclonal anti-GATA4 & ABCAM & Cat\# ab84593 \\
\hline Rabbit polyclonal anti- $\alpha$ SMA & ABCAM & Cat\# ab5694 \\
\hline Mouse monoclonal anti-V5 & ABCAM & Cat\# ab27671 \\
\hline Goat polyclonal anti-Brachyury & $\begin{array}{l}\text { Santa Cruz } \\
\text { Biotechnology }\end{array}$ & Cat\# sc-17743 \\
\hline Rabbit monoclonal anti-FoxA2 & ABCAM & Cat\# ab108422 \\
\hline Mouse monoclonal anti-Nestin & $\begin{array}{l}\text { Santa Cruz } \\
\text { Biotechnology }\end{array}$ & Cat\# sc-23927 \\
\hline Mouse monoclonal anti- $\alpha$ Tubulin-FITC & Merck & Cat\# F2168 \\
\hline Aanti-rabbit IgG-HRP & $\begin{array}{l}\text { Santa Cruz } \\
\text { Biotechnology }\end{array}$ & Cat\# sc-2357 \\
\hline Anti-mouse IgG-HRP & Cell Signaling Technology & Cat\# 7076 \\
\hline Donkey anti-goat IgG-Alexa fluor 555 & Thermo Fisher Scientific & Cat\# A-21432 \\
\hline Donkey anti-rabbit IgG-Alexa fluor 488 & Thermo Fisher Scientific & Cat\# A-21206 \\
\hline Goat anti-mouse IgG-Alexa fluor 488 & Thermo Fisher Scientific & Cat\# A-11029 \\
\hline
\end{tabular}

